\title{
Telemedizinische Methoden in der Hals-Nasen-Ohrenheilkunde
}

\section{Telemedical Methods in Otorhinolaryngology}

\section{(c) (i) (-) $\Theta$}

\author{
Autor \\ Achim G. Beule1, 2 \\ Institute \\ 1 HNO-Uniklinik Münster \\ 2 Klinik und Poliklinik für Hals-Nasen-Ohrenkrankheiten \\ der Universitätsmedizin Greifswald \\ Schlüsselwörter \\ Telemedizin, Kopf-Hals-Tumoren, Video-Otoskopie, \\ Palliativmedizin \\ Key words \\ Telemedicine, head and neck cancer, videootoscopy, \\ palliative care \\ Bibliografie \\ DOI https://doi.org/10.1055/a-0785-0252 \\ Laryngo-Rhino-Otol 2019; 98: S129-S151 \\ (c) Georg Thieme Verlag KG Stuttgart · New York \\ ISSN 0935-8943 \\ Korrespondenzadresse \\ Priv.-Doz. Dr. med. Achim G. Beule \\ Univ. HNO-Klinik \\ Kardinal-von-Galen-Ring 10 \\ D-48149 Münster
}

\section{ZUSAMMENFASSUNG}

Telemedizin ist ein Oberbegriff, der alle kommunikationstechnologisch unterstützte Verfahren zusammenfasst, die medizinische Leistungen über räumliche Entfernungen erbringen. Dieser rasch sich entwickelnde Bereich der Medizin besitzt gerade für das stark endoskopisch/mikroskopisch ausgerich- tete Fach der Hals-Nasen-Ohrenheilkunde interessante Optionen. Für die klinische Untersuchung von Ohr, Nase, Rachen und Larynx, aber auch für die postoperative Nachsorge und die Betreuung nach Kopf-Hals-Tumor-Erkrankungen liegen Daten zum telemedizinischen Vorgehen vor, ebenso für die audiologischen Messungen nach Cochlea-Implantation. Diese Daten bieten z. T. ein interessantes Potential, z. T. weisen sie aber gerade auch auf Probleme bei unkritischer Anwendung der Telemedizin hin, deren Erfolg entscheidend von der Ausbildung des Untersuchers vor Ort abhängt. Der vorgestellte Artikel fasst die wesentlichen Erkenntnisse dieses sich sehr rasch entwickelnden Forschungsgebietes überblickartig zusammen.

\section{ABSTRACT}

Telemedicine is a generic term summarizing all communication techniques assisting to provide medical services over distance. This rapidly developing medical area provides especially for the field of otorhinolaryngology with its high dependency on endoscopy and microcopy vaulable options. For clinical examination of ear, nose, throat and larynx, but also for postoperative care and surveillance of head and neck tumor patients data exist using the telemedical approach. Furthermore, audiological telemedical measurements even after Cochlear implantation are deemed feasible. While first data indicate interesting approaches, they also demonstrate problems, if a telemedical approach is used without proper preparation. Especially the observers at the remote location of the patients and their training are highly important in this regard. This review summarizes the current state of science of this rapidly changing are of research and clinical care.

\author{
Inhaltsverzeichnis \\ Redaktionelle Anmerkung \\ Einleitung und Hinweise zur Methodik \\ Definition \\ Historische Anfänge der Telemedizin \\ Nationale und internationale Rezeption der telemedizinischen \\ Entwicklungen
}

130

130

Rahmenbedingungen für den prinzipiellen Einsatz von telemedizinischen Maßnahmen
Telemedizinische Begrifflichkeiten 134

Ökonomische Aspekte der Telemedizin

Rahmenbedingungen für den sinnvollen Einsatz telemedizinischer Techniken

Faktoren, die die Verbreitung der Telemedizin behindern 136

Aspekte der Datenspeicherung 136

Aspekte des Schutzes von Privatdaten 136

Fernbehandlungsverbot 136

$\begin{array}{ll}\text { Weitere rechtliche Aspekte } & 137\end{array}$ 
Zusammenfassende Bewertung der rechtlichen Situation

Medizinische und technische Grenzen der Telemedizin

Akzeptanz telemedizinischer Methoden und Geräte in der Bevölkerung

Akzeptanz telemedizinischer Methoden in der Ärzteschaft

Krankheitsbilder und Indikationen

Gemischte Patientenkohorten

Postoperative Vorstellungen

Fachgleiche Netzwerke

Fachfremde Netzwerke

Daten aus homogenen Patientenkohorten

Otologie

Tele-Audiometrische Untersuchungen

Cochlear Implantation

Tinnitus

Neurootologie

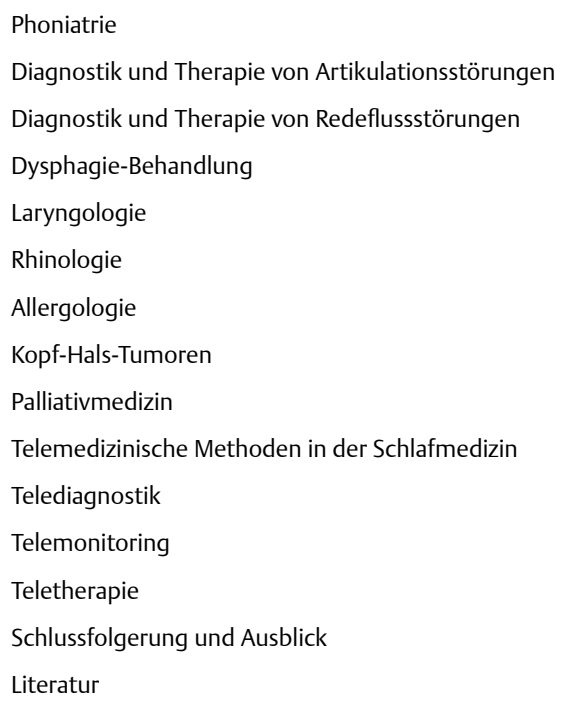

Diagnostik und Therapie von Artikulationsstörungen

Diagnostik und Therapie von Redeflussstörungen

Dysphagie-Behandlung

Laryngologie

Rhinologie

Allergologie

Kopf-Hals-Tumoren

Palliativmedizin

Telemedizinische Methoden in der Schlafmedizin

Telediagnostik

Telemonitoring

Teletherapie

Schlussfolgerung und Ausblick

Literatur

$\begin{array}{ll}\text { ABKÜRZUNGSVERZICHNIS } \\ \text { AGNES } & \begin{array}{l}\text { arztentlastende, gemeindenahe, } \\ \text { E-Health-gestützte, systemische } \\ \text { Intervention }\end{array} \\ & \text { Alaska Federal Health Care Access } \\ \text { AFHCAN } & \text { Network } \\ & \text { Gesetz für sichere digitale Kommunika- } \\ \text { E-Health-Gesetz } & \text { tion und Anwendungen im Gesund- } \\ & \text { heitswesen } \\ \text { ICC } & \text { Intraclass correlation coefficient } \\ \text { IT } & \text { Informationstechnologisch } \\ \text { MBO } & \text { Muster-Berufsordnung für Ärzte } \\ \text { MRSA } & \text { multi-resistenter Staphylokokkus aureus } \\ \text { odds Ratio (OR) } & \text { Chancenverhältnis } \\ \text { RFDS } & \text { Royal Flying Doctor Service } \\ \text { VERAH } & \text { Versorgungsassistentin in der Hausarzt- } \\ & \text { praxis } \\ 95 \% \text { CI } & \text { 95\% Konfidenzintervall }\end{array}$

\section{Redaktionelle Anmerkung}

Zur Verbesserung der Lesbarkeit wird die männliche Form von Substantiven verwendet, um Personen weiblichen, männlichen und sonstigen Geschlechtes gleichermaßen anzusprechen.

\section{Einleitung und Hinweise zur Methodik}

Zur Vorbereitung dieser Übersichtsarbeit über die Evidenzlage bei Durchführung einer telemedizinischen Methodik in verschiedenen Bereichen der Hals-Nasen-Ohrenheilkunde erfolgte eine systematische Literatursuche unter www.pubmed.com jeweils mit den Be- griffen „Telemedicine“, „eHealth“ und „telehealth“ in Verbindung mit Begriffsbezeichnungen für die einzelnen Organbereiche und den häufigsten Diagnosen des Fachgebietes. Zusammenfassungen wurden gesichtet und bei Angabe relevanter Daten im Volltext ausgewertet. In zahlreichen Publikationen zeigten sich insbesondere ältere Arbeiten aus nicht in pubmed.com gelisteten Journalen, die auf der Basis einer Einzelfallentscheidung ebenfalls im Volltext ausgewertet wurden, sofern dieser zugänglich war.

Ergänzt wurde die Beurteilung der Sachlage durch das Studium juristisch einschlägiger Nachschlagewerke und Internetseiten wie juris.de und von Kommentaren in Zusammenhang mit aktuell im Internet publizierten Texten, speziell zu den Beschlüssen des Deutschen Ärztetages.

Aufgrund der Heterogenität der aufgefundenen Literaturquellen kann der vorliegende Text schon aus rein methodischen Gründen den Anspruch auf Vollständigkeit nicht erbringen. Um eine möglichst objektive Darstellung zu erreichen, wurde auf die Nennung beteiligter Personen weitestgehend verzichtet. Stattdessen wurden wesentliche organisatorische Charakteristika der genannten Telemedizin-Netzwerke in den Vordergrund gestellt, um so die Vergleichbarkeit zu verbessern.

Nach Darstellung der verschiedenen Definitionen der „Telemedizin" schließen sich zum besseren Verständnis Angaben zur geschichtlichen Entwicklung und zur ökonomischen Bedeutung an. Da die Etablierung telemedizinischer Methoden innerhalb der HalsNasen-Ohrenheilkunde sehr heterogen ist, werden zunächst Faktoren, die die Entwicklung von entsprechenden Netzwerken begünstigen oder verhindern einschließlich rechtlicher Rahmenbedingungen vorgestellt, bevor die Studienlage, geordnet nach Organbereichen und den häufigsten Diagnosen des Fachgebietes, präsentiert und diskutiert wird. 


\section{Definition}

Telemedizin ist gemäß Definition der Bundesärztekammer „ein Sammelbegriff für verschiedenartige ärztliche Versorgungskonzepte, die als Gemeinsamkeit den prinzipiellen Ansatz aufweisen, dass medizinische Leistungen der Gesundheitsversorgung der Bevölkerung in den Bereichen Diagnostik, Therapie und Rehabilitation sowie bei der ärztlichen Entscheidungsberatung über räumliche Entfernungen (oder zeitlichen Versatz) hinweg erbracht werden. Hierbei werden Informations- und Kommunikationstechnologien eingesetzt. “ [1, 2] (vgl. > Infobox 1). Die Anfänge der Telemedizin beruhen auf dem weltweiten Problem, möglichst allen Menschen einen adäquaten Zugang zu Leistungen des Gesundheitssystems zu gewährleisten. Das Institut für Medicine [3] definierte für die USA Telemedizin als „Gebrauch elektronischer Informations- und Kommunikationstechnologien, um das Gesundheitswesen zu unterstützen und den Zugang zu ermöglichen, wenn Distanz die Teilnehmer trennt“. Dabei wird der alleinige Gebrauch von Telefon oder Fax, z. B. im Rahmen der Abrechnung durch Medicaid, nicht als Telemedizin bezeichnet.

Telemedizin ist ein Sammelbegriff für verschiedenartige ärztliche Versorgungskonzepte, die als Gemeinsamkeit den prinzipiellen Ansatz aufweisen, dass medizinische Leistungen der Gesundheitsversorgung der Bevölkerung in den Bereichen Diagnostik, Therapie und Rehabilitation sowie bei der ärztlichen Entscheidungsberatung über räumliche Entfernungen (oder zeitlichen Versatz) hinweg erbracht werden. Hierbei werden Informations- und Kommunikationstechnologien eingesetzt.

Historisch steht damit die räumliche Distanz im Vordergrund, sowie Hindernisse wie in der See- [4-6] Luft- und Raumfahrt [7], und damit eine Barriere, die es zwischen Arzt und Patient oder zwischen Allgemeinarzt und Spezialisten zu überbrücken gilt. Deshalb wundert es nicht, dass wegen der großen Entfernungen in Australien die Telemedizin ihren Ursprung hatte und später aus Alaska weitere Impulse kamen.

\section{Historische Anfänge der Telemedizin}

1928 gründete der Pfarrer John Flynn von der Presbyterianischen Kirche den Aerial Medical Service mit dem Ziel, die medizinische Versorgung im australischen Outback zu verbessern [https://www. flyingdoctor.org.au/]. Bis zu diesem Zeitpunkt waren für das mehrere Millionen Quadratkilometer große Areal nur 2 Ärzte verfügbar. Entsprechend versorgten sich die australischen Farmer ebenso wie die Einwohner Alaskas zu diesem Zeitpunkt in erster Linie mit Hilfe von Naturheilmitteln. Aufgrund der starken kulturellen Kluft zwischen den Eingeborenen (Aborigines) und den Einwanderern war das Ausmaß, in dem Schamanentum angenommen wurde, in Australien deutlich geringer als in Alaska.

In Alaska waren aufgrund seiner militärischen Bedeutung während des 2. Weltkrieges Krankenschwestern in 14 Militärbasen stationiert. Nach Kriegsende blieben diese weiterhin in den Städten und verbesserten damit bereits die medizinische Versorgungssituation [8]. Außerdem wurde u. a. ab 1945 eine mobile Untersuchungseinheit mit einem ausgemusterten Militär-LKW geschaffen. Sie bot so erstmals die Möglichkeit auch unzugängliche und ländliche Gegenden mit medizinischer Ausrüstung aufzusuchen. Ergänzt wurde diese Maßnahme ab 1946 durch ein medizinisch ausgestattetes Schiff, mit dem auf dem Wasserweg Küstensiedlungen
Telemedizin ist ein Sammelbegriff für verschiedenartige ärztliche Versorgungskonzepte, die als Gemeinsamkeit den prinzipiellen Ansatz aufweisen, dass medizinische Leistungen der Gesundheitsversorgung der Bevölkerung in den Bereichen Diagnostik, Therapie und Rehabilitation sowie bei der ärztlichen Entscheidungsberatung über räumliche Entfernungen (oder zeitlichen Versatz) hinweg erbracht werden. Hierbei werden Informations- und Kommunikationstechnologien eingesetzt.

- Infobox 1 Definition der Telemedizin durch die Bundesärztekammer [2]

versorgt wurden. Mit fortschreitender Industrialisierung, dem Bau von Eisenbahnen und der Erschließung größerer Siedlungen wurden diese mobilen Einheiten durch ein weiteres Schiff und Einheiten auf Eisenbahnzügen bis in die 1950er Jahre hinein ergänzt.

In Australien wurden als erste Schritte Buschkrankenhäuser gegründet, aber auch diese konnten das Problem der enormen Entfernungen nicht überbrücken. Zudem behinderte das Klima die Erschließung des Landes durch Straßen. Vor diesem Hintergrund setzte Pfarrer John Flynn auf die durch die technischen Entwicklungen im 2. Weltkrieg deutlich zuverlässiger gewordenen Flugzeuge, um medizinisches Personal zu transportieren [9].

Der erste Flughafen und Stützpunkt wurde am 15. Mai 1928 in Cloncurry (Queensland) eröffnet [10]. Weitere Stützpunkte folgten in Charters Towers und Charleville, später in Alice Springs (1939) und dann auch in Südaustralien (Port Augusta und Adelaide) und dem Northern Territory (Yulara). Der Dienst änderte seinen Namen ab 1942 in Flying Doctor Service, ab 1955 in Royal Flying Doctor Service (RFDS; kurz The Flying Doctors). Bis heute sind dabei die Farmer vor Ort verpflichtet, die Landebahn (meist ein Stück Straße in der Nähe der Farm) von Pflanzen und Steinen zu befreien, um dem RFDS eine gefahrlose Landung zu ermöglichen.

Parallel war sowohl in Alaska, als auch in Australien, die Entwicklung von Telekommunikationsapparaten erforderlich, um medizinische Hilfe und Rat anzufordern. In Alaska wurden zunächst konventionelle Telegramme benutzt, dann auch Radio- und Funkgeräte. In Australien war die Verbesserung der medizinischen Versorgung eng mit der Entwicklung eines vom Stromnetz unabhängigen Funkgerätes verbunden. Bis zu einer Reichweite von mehr als 500 km konnten die Farmer mittels Pedalantrieb mit einer Zentrale Kontakt aufnehmen. Damit begann auch zugleich die Ära der telemedizinischen Beratung in Australien: So wurden und werden bis heute bei Bewohnern des Outback Kisten mit Notfall-Medikamenten deponiert, deren Inhalt nach Rücksprache mit dem RFDS zum Einsatz kommt. Derzeit sind 2338 derartige Kisten im gesamten Outback verteilt und helfen, den Zeitverzug bis zum Eintreffen von qualifizierter Hilfe zu verkürzen. So wird anhand eines standardisierten Körperschemas Art und Lokalisation von Beschwerden am Funkgerät der Zentrale mitgeteilt, die daraufhin ggf. sinnvolle Medikamente, nach Nummern bezeichnet, empfiehlt.

Insgesamt wird vom RFDS derzeit ein Gebiet von etwa 7,69 Millionen $\mathrm{km}^{2}$ bedient, was in etwa 2 Dritteln der Gesamtfläche Australiens entspricht [https://www.flyingdoctor.org.au/about-therfds/]. Allein der Stützpunkt in Alice Springs ist heute für ein Gebiet von 1,25 Millionen $\mathrm{km}^{2}$ mit einem Radius von $600 \mathrm{~km}$ zuständig, in 
dem etwa 16000 Menschen leben. Pro Jahr werden etwa 26 Millionen Flugkilometer absolviert, und z. B. im Jahr 2016/17 knapp 37000 Australier transportiert. Der RFDS ist damit bis heute ein wichtiger Baustein des australischen Gesundheitswesens und hat sich einen festen und anerkannten Platz erobert. Dieser wird auf der australischen 20 Dollar-Note durch das Bild des Gründers des RFDS, neben Flugzeug und Funkgerät, veranschaulicht.

In Alaska wurde dagegen aufgrund verschiedener, auch wirtschaftlicher, Entwicklungen der Bedarf abseits der großen Siedlungen in den 1960-1990er Jahren deutlich geringer, so dass man die mobilen Einheiten abschaffte. Im Verlauf der wirtschaftlichen Rezension wurden aber Probleme, besonders beim Missbrauch von Substanzen und in der Psychiatrie, offensichtlich. Diese Erkenntnis förderte eine Änderung in der Grundausrichtung der Telemedizin: Es ging nun nicht mehr darum, den Arzt zum Patienten zu bringen, sondern den Kontakt zwischen beiden herzustellen, um unnötige Reisen ggf. zu vermeiden.

Das heutige Ergebnis ist das Alaska Federal Health Care Access Network (AFHCAN). Dies ist eine Stammesorganisation, die mehr als 200 verschiedene Standorte miteinander telemedizinisch verbindet und bereits seit 2001 besteht [11]. Das besondere Problem in Alaska war lange die eingeschränkte Datenübertragungsrate. Deshalb waren zunächst gespeicherte Texte, Bilder und Datensätze versendet worden. Erst in den letzten Jahren konnte das Angebot um Live-Videokonferenzen erweitert werden, die gesichert und verschlüsselt gesendet werden. Derzeit bearbeitet das Netzwerk ca. 3,000 Fälle pro Jahr, von denen ca. 2/3 am Tag der Anfrage bearbeitet werden und sogar $50 \%$ innerhalb von einer Stunde. Etwa ein Viertel beziehen sich auf Anfragen an Spezialisten, 75\% auf Allgemeinärzte. Für die HNO-Heilkunde liegen aus Alaska Daten vor, dass die Wartezeit durch den Einsatz von Telemedizin deutlich verkürzt werden konnte. So warteten früher $47 \%$ der Patienten 5 Monate oder mehr auf einen Termin beim HNO-Arzt, seit Einsatz der Telemedizin sank diese Rate zunächst auf $8 \%$ und mittlerweile auf $3 \%$ [12]. Dank eines integrierten Video-Otoskopes wird in Alaska Telemedizin auch zur Planung von Ohreingriffen und zur otologischen Nachkontrolle $[13,14]$, aber zunehmend auch im übrigen HNO-Gebiet eingesetzt [15]. So wurde die Notwendigkeit zum Arzt zu reisen für 75\% der Patienten mit geplanter Vorstellung beim Spezialisten bzw. 25\% der geplanten Vorstellung beim Allgemeinarzt vermieden.

Die verbesserte Integration dieser sehr ländlichen Gegenden zeigte den großen Unterschied in der Gesundheitsversorgung im Vergleich zu den großen Städten. So ist nicht nur der Zugang zu ärztlicher Hilfe im ländlichen Raum erschwert, sondern chronische Erkrankungen sind in abgelegenen Gegenden auch deutlich häufiger. Hier erwies sich Alaska mit seinen „Mental Health“ Programmen als Vorreiter, um Aufmerksamkeit auf die Folgen der Isolation in unwirtlichen Gegenden zu lenken.

Die technische Weiterentwicklung der auf diese Weise gewonnenen Erkenntnisse erlaubt mittlerweile die „real-time“ Analyse der Gesundheitsdaten von Astronauten im All seit dem Beginn der Space Shuttle Ära der NASA [7].

\section{Nationale und internationale Rezeption der telemedizinischen Entwicklungen}

Vor dem Hintergrund der oben aufgeführten technischen Entwicklungen präsentierte sich die Telemedizin weltweit als Option, Pro- bleme der Gesundheitsfürsorge in ländlichen Regionen zu verbessern gerade auch in sich erst entwickelnden Gebieten von Afrika und Asien [16].

Vor diesem Hintergrund nannte der Weltärztebund 2006 die Telemedizin als eine Möglichkeit, um Patiententransporte zu vermeiden [16]. Bereits im folgenden Jahr wurden die Anforderungen an die Telemedizin durch den Weltärztebund präzisiert [17]. Auch wenn der Titel dieser Publikation eine ethische Dimension suggeriert, wurden hier erstmals wesentliche inhaltliche Aspekte verbindlich festgestellt. Hinzu kam eine erste Definition der Telemedizin als eine Technik, Medizin über eine räumliche Distanz auszuüben und dabei Interventionen, diagnostische Vorgänge und Behandlungsentscheidungen durchzuführen. Der Weltärztebund sah Telemedizin zu diesem Zeitpunkt primär in einer Situation, in der Arzt und Patient sich schon von einem früheren persönlichen Kontakt kennen. Jedoch wurden bereits die Verpflichtung zur detaillierten Dokumentation und Datensicherheit, die Möglichkeit zur gegenseitigen Identifikation und möglichst auch Interaktion der Beteiligten, die Notwendigkeit zur Patienteninformation und -aufklärung, zur Qualitätskontrolle, zur Kosteneffizienz und zum Evidenz-basierten-Vorgehen benannt und gefordert. Entsprechend besitzt dieses Dokument bis heute Gültigkeit. Dabei stellte der Weltärztebund explizit fest, dass sich telemedizinische Techniken immer an den lokalen Gegebenheiten orientieren sollen, um so den heterogenen Bedürfnissen weltweit Rechnung zu tragen.

2010 hat die Bundesärztekammer darauf aufbauend Leitsätze für Deutschland verfasst [18], um die rasant fortschreitenden Entwicklungen auf dem Gebiet der Telemedizin für Deutschland zu begleiten und ärztlich zu steuern. Dazu wurde die Entschließung „Erste Voraussetzungen für gute Telemedizin“ anlässlich des 113. Deutschen Ärztetages gefasst. Sie forderte vor dem Hintergrund vieler projektbasierter Insellösungen einen Mehrwert in der Patientenversorgung und sprach das Telemonitoring von Patienten mit chronischen Erkrankungen an, um die Qualität der Patientenversorgung zu verbessern. Telemonitoring dient durch wiederholte Messung des gleichen Parameters (z. B. morgendliches Gewicht bei bekannter Herzinsuffizienz) der Früherkennung und Intervention (z. B. durch Aufforderung zur Einnahme einer zusätzlichen Medikation, hier z. B. Furosemid), um so Krankenhausaufenthalte zu vermeiden. Untersuchungen in verschiedenen Fachbereichen zeigen, dass Patienten diese Form der engmaschigen Begleitung sehr begrüßen, da Krankenhausaufenthalte und Arztbesuche entfallen können [19, 20]. Telemedizin wurde also bereits 2010 als sinnvolle Möglichkeit angesehen, um mittels Telekonsultation auch in Deutschland räumliche Barrieren zu überwinden. Als Königsbeispiel darf der Aufbau von Netzwerken zur Früherkennung und -behandlung von Schlaganfällen gelten [21-24]. Im gleichen Dokument wurden erste Anforderungen an die Qualität der Telemedizin gestellt mit einem klaren Votum gegen arztersetzende, also substitutive Prozesse.

\section{Rahmenbedingungen für den prinzipiellen Einsatz von telemedizinischen Maßnahmen}

Gerade in den Anfängen der Telemedizin mangelte es oftmals an der Akzeptanz der beteiligten Ärzte, Behandler anderer Gesundheitsprofessionen und der Patienten. So bestanden Vorbehalte ge- 
genüber der Zuverlässigkeit der Telemedizin im Vergleich zum konventionellen Arzt-Patientenkontakt. Dabei ist auch nach Ansicht der Bundesärztekammer [18] Telemedizin und konventionelle Medizin kein Gegensatz, sondern eine Ergänzung. So sollen telemedizinische Anwendungen ärztliches Handeln unterstützen, bei Beibehaltung gleicher Qualitäts- und Dokumentationsstandards.

Anfänglich wurde die Anwendung telemedizinischer Verfahren nur dann angeraten, wenn konventionelle Methoden aufgrund der spezifischen Anforderungen im Einzelfall (z. B. der Qualifikation der Mitarbeiter oder der technischen Ausrüstung), der Verhältnisse des Ortes (aufgrund z. B. der räumlichen Entfernung) oder der Zeit der Inanspruchnahme nicht verfügbar waren. Auch für die telemedizinische Versorgung gilt dabei der Grundsatz eines Facharztstandards. Daher wurden auch hier für die Durchführung qualitätssichernde Maßnahmen gefordert.

Darüber hinaus setzt die Anwendung von Telemedizin Vorkenntnisse von Arzt und Patient voraus. Insbesondere ist es erforderlich, dass diese mit den heutigen technischen Hilfsmitteln Smartphone, Tablet oder Computer bzw. einem Medizinprodukt hinreichend vertraut und sicher sind, um die Aufmerksamkeit zum eigentlichen
Gegenstand der Gesundheitsfürsorge zu lenken. Der Arzt muss eine ausreichende fachliche Kompetenz für die medizinische Fragestellung, wie auch für das medizinische Verfahren besitzen. Er sollte über Kenntnisse von technischen und inhaltlichen Abläufen inklusive möglichen Komplikationen, aber auch der Dokumentation, verfügen. Für den Fall eines Telekonsils, also einer Verständigung von Arzt zu Arzt, sollte der zugezogene Konsiliarius (Spezialist) ausreichende Kenntnisse über die Ausstattung und Arbeitsbedingungen des Kollegen besitzen, um entsprechend sinnvoll und umsetzbar Hilfestellung leisten zu können.

Vor jeder telemedizinischen Behandlung muss der Patient umfassend aufgeklärt werden, speziell auch über die möglichen Nachteile einer rein telemedizinischen Untersuchung, damit dieser ihr zustimmen kann. Dies gilt in besonderem Maße, wenn alternativ auch eine persönliche Vorstellung prinzipiell möglich wäre. Der Patient soll so befähigt werden, selbst die ggf. in Kauf zu nehmende Mehrbelastung in Relation zu der durch die Grenzen der telemedizinischen Methodik bedingten Unsicherheit abzuschätzen. Ferner ist sein Einverständnis auch unter dem Aspekt des Datenschutzes

\section{Anwendungsart:}

- Telediagnostik: Übersendung von Untersuchungen zur Befundung

- Telekonsil: Arzt-zu-Arzt-Kontakt, meist Allgemeinmedizin mit Spezialist

- Telekonsultation: Patient-zu-Arzt-Kontakt

- Telemonitoring: wiederholte Übermittlung derselben Untersuchungsergebnisse

- Teletherapie: therapeutische Intervention über einen telemedizinischen Kontakt

\section{Zeitliche Dimension:}

- zeitversetzt /asynchron: Übersendung gespeicherter Daten

- zeitgleich / live: Echtzeit Verbindung (z.B. Videokonferenz)

\section{Beteiligte:}

- Arzt-zu-Arzt: Telekonsil oder zur Weiterbildung von Ärzten

$\circ$ fachfremd / fachgleich

- Arzt-zu-Patient: Telekonsultation, z.B. um Reisen bei Nachkontrollen zu vermeiden

- Patient-zu-Patient: Erfahrungsaustausch und Beratung z.B. im Rahmen von Selbsthilfegruppen

\section{Ort der Durchführung:}

- Verbindung zum Ort des Patienten (Häuslichkeit, Schule, Heim)

- Patient begibt sich zu einem telemedizinischen Untersuchungszentrum (z.B. in eine heimatnahe Praxis, Krankenhaus oder Schule).

- Infobox 2 Taxonomie telemedizinischer Maßnahmen (modifiziert nach [2, 25-27]). 
für die Speicherung der Daten und deren Übermittlung, Bewertung und Dokumentation erforderlich.

\section{Telemedizinische Begrifflichkeiten}

Aufgrund der raschen Entwicklung der Telemedizin in den letzten Jahren fehlt eine einheitliche und klare Bezeichnung (Taxonomie), obwohl verschiedenen Autoren [25, 26]- darunter auch 2015 die Bundesärztekammer [2] mit der Arbeitsgemeinschaft Telemedizin - sich um eine Einordnung bemüht haben.

Der damals verwendete Begriff „eCare“ der Bundesärztekammer hat sich nicht durchgesetzt, er wird heute umgangssprachlich häufig durch „Telemedizin“ oder auch „eHealth“ ersetzt. Dabei war eHealth ursprünglich als übergeordneter Terminus aller technologischen Vorgänge mit dem Hintergrund von Gesundheitsfragen definiert worden. Telemedizin wird als ein Anwendungsfeld [27] von eHealth benannt und abgegrenzt von ähnlichen Technologien, die z. B. für Forschungs- oder administrative Zwecke eingesetzt werden [2].

Prinzipiell sind telemedizinischen Maßnahmen nach der zeitlichen Art der Datenübertragung, den beteiligten Personen, der Vorstellungsart des Patienten, aber auch der Funktionalität, dem Applikationsmodus und der Technologie differenzierbar. Die Technologie soll in diesem Hauptreferat nicht behandelt werden wegen der Heterogenität und des schnellen Wandels. Auf die Wichtigkeit von Datenschutz und Datenqualität für eine verlässliche und sichere Übertragung, sei hier aber erneut hingewiesen. \ Infobox $\mathbf{2}$ gibt einen Überblick über die relevanten taxonomischen Begriffe, um die im Folgenden charakterisierten Anwendungsbeispiele entsprechend zuordnen zu können und auch nach eigenen Vorstellungen zu vergleichen.

Übereinstimmend mit der Entwicklung auch nach 2015 werden dabei die unterschiedlichen Anwendungsarten gegeneinander abgegrenzt. Die häufigsten sind Telediagnostik, Telekonsil, Telekonsultation, Telemonitoring und Teletherapie. Da diese prinzipiell in jedem medizinischen Fachgebiet ablaufen und auch Gebietsgrenzen überschreiten können, plädierte die AG Telemedizin der Bundesärztekammer schon 2015 für die Verwendung des Begriffs „telemedizinischen Maßnahmen“ statt „Telemedizin“. Dadurch sollte der Eindruck eines sich entwickelnden, eigenständigen medizinischen Gebietes vermieden werden [2].

Im Rahmen der berufspolitischen Diskussion um telemedizinische Methoden wurde im Bereich der Beteiligten unterschieden, inwieweit die telemedizinisch verbundenen Ärzte fachgleich arbeiten, d. h. beide im gleichem Gebiet, wie z. B. der HNO-Heilkunde, tätig sind oder ob einer fachfremd ist, also wenn z. B. ein Allgemeinmediziner HNO-ärztliche Untersuchungen durchführt und die Befunde einem HNO-Arzt zusendet.

\section{Ökonomische Aspekte der Telemedizin}

Telemedizin stellt einen stark wachsenden Markt dar, der aufgrund der rasanten Entwicklungen, auch wirtschaftlich gesehen, sehr attraktiv ist. Dazu tragen zunehmend Daten bei, die telemedizinischen Maßnahmen eine Kosteneffizienz und Einsparungspotenzial gegenüber traditionellen Verfahren bescheinigen [28]. Diese führen in den USA zunehmend dazu, dass der (Bundes-)Staat Kosten für telemedizinische Leistungen trägt. So übernimmt der Staat IIlinois als 49. Bundesstaat der USA ab 2018 innerhalb von Medicaid die Kosten für telemedizinische Leistungen in Form von Live Videos. 15 Bundesstaaten übernehmen Kosten bei zeitversetzter Aufnahme und Versendung von Daten, 20 Bundesstaaten finanzieren Telemonitoring von Patienten und weitere neun Bundesstaaten übernehmen Kosten von allen 3 technischen Vorgängen. Zusätzlich sind in 32 Bundesstaaten eine Übertragungsgebühr oder eine Gerätepauschale abrechenbar (Mehrfachnennungen möglich) [29]. Der Vorschlag zur Kostenübernahme in den USA nicht nur für Medicaid (Nach Bundesstaaten organisierte Kostenübername für Kinder) sondern auch für Medicare (vom Bund organisierte Kostenübernahme für erwachsene Patienten) wird derzeit beraten - ein Beschluss wird für 2019 erwartet [30].

Derartige Entwicklungen tragen dazu bei, dass die weltweite Wachstumsrate für den telemedizinischen Markt auf jährlich 27.5\% bis 2021 geschätzt wird [31]. Dabei besitzen die USA die ökonomisch größte Bedeutung, gefolgt von Europa und Asien.

Außerhalb der USA hinkt die Finanzierung telemedizinischer Netzwerke noch deutlich der technischen Entwicklung hinterher [32]. Die bislang von Krankenkassen in Deutschland in Selektivverträgen und Modellprojekten unterstützten telemedizinischen Anwendungen finden sich überwiegend im Bereich des Telemonitoring [33]. Das Gesetz für sichere digitale Kommunikation und Anwendungen im Gesundheitswesen (E-Health-Gesetz) vom 21.12.2015 [34] stellte erstmals die Finanzierung von Videokonferenzen in Aussicht. Nach Konkretisierung der technischen Rahmenbedingungen [35] wurde auch die Finanzierung der Videosprechstunde zum 01.April 2017 beschlossen. Demnach können die in $~ I$ Infobox 3 benannten Facharztgruppen und damit auch Hals-Nasen-Ohrenärzte bei den in \ Infobox 4 aufgeführten Indikationen Videosprechstunden als Vertragsarzt durchführen. Eine Ausweitung der Indikationsliste ist bereits geplant.

Voraussetzung ist jeweils der Nachweis, dass ein zertifizierter Videoanbieter eingebunden ist in die technische Übertragung. Die Zertifizierungen laufen und können aktuell unter http://www.kbv. $\mathrm{de} / \mathrm{html} / \mathrm{videosprechstunde.php} \mathrm{nachvollzogen} \mathrm{werden.}$

Die Vergütung der Videosprechstunde besteht aus 2 Komponenten [37]: Pro Sprechstunde erhält der durchführende Arzt einen Technikzuschlag von 4,21 Euro. Die Anzahl der Videosprechstunden ist auf insgesamt 50 Mal im Quartal gedeckelt. Falls der Patient in einem Quartal nicht die Praxis aufsucht, können Ärzte einmal im Behandlungsfall 9,27 Euro erhalten. Voraussetzung ist, dass der Patient in den vorangegangenen 2 Quartalen mindestens einmal in der Praxis persönlich vorstellig war und die Verlaufskontrolle durch dieselbe Praxis erfolgt wie die Erstbegutachtung. Aufgrund der geschätzten Lizenzgebühren für Videodienste von etwa 100 Euro im Quartal wird seitens der Kassenärztlichen Bundesvereinigung prognostiziert, dass die Videosprechstunde ab einem Aufkommen von 2 Patientenkontakten pro Woche für einen Vertragsarzt kostendeckend ist.

Zusammenfassend ist damit festzustellen, dass erstmals auch in der deutschen Regelversorgung für den HNO-Arzt die Abrechnung telemedizinischer Leistungen möglich ist. Ob die Einrichtung einer Videosprechstunde als Betreuungsinstrument des HNO-Vertragsarztes ökonomisch sinnvoll ist, muss für jeden Arzt individuell beurteilt werden. Das Ergebnis jeder Kosten-Nutzen-Analyse wird 
- Hausärzte

- Kinder-und Jugendärzte

- Anästhesisten

- Augenärzte

- Chirurgen

- Hals-Nasen-Ohrenärzte

- Mund-, Kiefer- und Gesichtschirurgen

- Neurologen, Nervenärzte und Neurochirurgen

- Orthopäden

- Gynäkologen

- Dermatologen

- Fachärzte für Innere Medizin

- Psychiater

- Urologen

- Phoniater und Pädaudiologen

- Fachärzte für physikalische und rehabilitative Medizin

- Strahlentherapeuten

> Infobox 3 Facharztgruppen, die eine Videosprechstunde durchführen und abrechnen dürfen (Stand 09/2018) [36]

Videosprechstunden können bei folgenden Anlässen durchgeführt werden:

- Visuelle postoperative Verlaufskontrolle einer Operationswunde

- Visuelle Verlaufskontrolle einer/von Dermatose(n), auch nach strahlentherapeutischer Behandlung

- Visuelle Verlaufskontrolle einer/von akuten, chronischen und/oder offenen Wunden

- Visuelle Beurteilung von Bewegungseinschränkungen/-störungen des Stütz-und Bewegungsapparates, auch nervaler Genese, als Verlaufskontrolle

- Beurteilung der Stimme und/oder des Sprechens und/oder der Sprache als Verlaufskontrolle

- Anästhesiologische, postoperative Verlaufskontrolle

Infobox 4 Indikationsliste für Videosprechstunden (Stand 09/2018) [36]

abhängen von der örtlichen Akzeptanz einerseits, der Kostenentwicklung für die zertifizierten Videoanbieter andererseits, der Kosten für die Anschaffung und Unterhaltung der Infrastruktur für eine Videosprechstunde und der lokalen Konkurrenzsituation. Die Tatsache, dass die benachbarte Facharztgruppe der Mund-Kiefer-Gesichtschirurgie hier zur Durchführung ebenfalls berechtigt wurde, mag den Wettbewerb auch im digitalen Bereich intensivieren.

\section{Rahmenbedingungen für den sinnvollen Einsatz telemedizinischer Techniken}

Häufig wird der Einsatz telemedizinischer Methoden aus ökonomischer Sicht durch die mögliche Einsparung von Reisekosten gerechtfertigt. Dabei ist dies nur einer der Gründe für eine zweckmäßige Anwendung dieser modernen Technik:

Vorteile der Telemedizin werden v. a. für ländliche Regionen gesehen $[38,39]$, besonders in schlecht entwickelten Regionen der Welt, in denen aus wirtschaftlichen Gründen die Arztdichte gering ist [39]. Vermehrte Untersuchungen liegen für Kriegsveteranen [40] vor, da allgemein höheres Lebensalter bzw. assoziierte Komorbiditäten die Vermeidung einer Fahrt zur ärztlichen Untersuchung attraktiv werden lässt. Dabei spielt auch die Finanzierung der ärztlichen Untersuchung eine Rolle: Da bspw. in den USA die Veteran Affairs die Fahrtkosten der Patienten begleichen müssen, liegt hier ein starker Anreiz vor, Fahrten zu vermeiden [40]. So konnten 145 Meilen und 142 min pro Vorstellung bzw. \$18,555 pro Jahr eingespart werden. Inhaltlich wurden Fächer, die ein hohes Maß an ArztPatienten-Interaktion bedürfen, als besonders geeignet für die Telemedizin bezeichnet [41]. Zu beachten ist dabei, dass weniger Fahrten nicht nur die Patienten (bzw. bei Kostenübernahme öffentliche Geldgeber) entlasten, sondern auch die Umweltverschmutzung durch Reduktion z. B. des Kohlenstoffdioxidausstosses senkt.

Ein Vergleich der telemedizinischen Techniken zeigt, dass diese v. a. unter Verwendung optischer Geräte (Endoskope, Mikroskope, Kameras) eingesetzt werden [41]. Dabei spielt die Datenqualität der optischen Geräte (Auflösung, Anzahl Bilder pro Sekunde) eine wichtige Rolle [41].

Zeitversetzte Befundung von Untersuchungen sowie Unterstützung in der Terminplanung inkl. -erinnerung kann hilfreich sein, vorhandene Ressourcen besser auszunutzen.

Die Überwindung von Barrieren ist aber nicht nur räumlich auf die überbrückten Wege zu beziehen. Auch der erhöhte Aufwand bei der Vorstellung von Patienten, deren Transport sehr aufwendig ist (z. B. von solchen, die mit multi-resistenten Keimen wie multiresistentem Staphylokokkus aureus [MRSA] besiedelt sind, Intensiv- oder Schwerlasttransporte, Gefängnisinsassen) kann so verringert werden. Das gleiche gilt bei erschwertem Zugang des bettlägerigen oder pflegebedürftigen Patienten (z. B. als Heimbewohner) zur Gesundheitsfürsorge. Dies gewinnt vor dem Hintergrund des demografischen Wandels mit der Zunahme des Anteils von Menschen im Alter ab 65 Jahren auf dann $32 \%$ im Jahre 2060 [42] weiter an Bedeutung. Auch die Angehörigen gesellschaftlicher Randgruppen [43] wie amerikanische oder australische Ureinwohner können auf diesem Wege besser medizinisch versorgt werden.

Die Verbesserung der medizinischen Versorgung [44] gelingt mit Hilfe telemedizinscher Methoden primär durch Überbrückung sektoraler Grenzen (z. B. durch einfachere Hinzuziehung von Spezialisten) mit Austausch aller verfügbaren Informationen über den Patienten. Dieser kann zugleich seine Sicht der Beschwerden, ein vor Ort tätiger Kollege etwaige umfangreiche Kenntnisse der medizinischen Vorgeschichte des Patienten und der Tele-Konsiliar das aktuelle Fachwissen in die Behandlungsplanung einfließen lassen. Dies erhöht die Transparenz des Leistungs- und Behandlungsgeschehens (Patientensouveränität) und ermöglicht dem Patienten so die aktive Teilnahme am Gesundheitswesen. 
Aufgrund des stark strukturierten Ablaufes in der telemedizinischen Versorgung mit digitaler Bereitstellung der Daten wird die Auswertung der erhobenen Daten für Forschungszwecke, wie speziell der Versorgungsforschung und der Gesundheitsberichterstattung für die Verbesserung der Versorgungsoptionen, einfacher nutzbar. - Infobox $\mathbf{5}$ stellt als Checkliste die genannten Faktoren zusammen, um eine Versorgungssituation oder ein telemedizinisches Projekt auf eine sinnvolle Konzeption hin prüfen zu können

\section{Faktoren, die die Verbreitung der Telemedizin behindern}

\section{Rechtliche Aspekte}

Immer wieder werden ausführlich rechtliche Aspekte diskutiert, die der Anwendung telemedizinscher Techniken entgegen stehen [45-47]. Aufgrund der derzeitigen Entwicklung im Zusammenhang mit dem E-Health-Gesetz vom 21.12.2015 [34], aber auch wegen aktueller Beschlüsse zur Lockerung der Fernbehandlung und noch fehlender Rechtsprechung kann an dieser Stelle - neben mangelnder juristischer Qualifikation des Autors - keine abschließende Einschätzung gegeben werden. Allerdings sollen einige aus Sicht des telemedizinisch tätigen Arztes relevante Problemfelder benannt werden, selbstverständlich ohne dabei eine Art von Rechtsberatung anzustreben.

Die für einen telemedizinisch tätigen Arzt wichtigen Problemfelder betreffen:

- große Entfernung/geographische HIndernisse zwischen Arzt und Patient (z.B. Versorgung auf See, während des Fluges, in ländlichen Regionen)

- eingeschränkte Mobilität des Patienten z.B. durch Bettlägerigkeit, Beatmungspflichtigkeit, Bedarf an intensivmedizinischer Betreuung, Besiedlung mit Multiresistenten Keimen, extremes Übergewicht, Ko-Morbiditäten, Überwachungspflicht (Gefängnisinsassen)

- Erleichterter Zugang für den Patienten zu Leistungen des Gesundheitswesens, z.B. für gesellschaftliche Randgruppen

- vorliegende Evidenz für eine Vergleichbarkeit von telemedizinscher und konventioneller Betreuung

- Vorliegen einer medizinischen Notfallsituation, in der eine telemedizinische Beurteilung schneller erfolgen kann als eine konventionelle Vorstellung

- Ambulanter Charakter des Arzt-Patienten-Kontaktes

- Beteiligung spezialisierter Zentren / Fachärzte/ teurer Technik

- Sektorenübergreifender Charakter einer Vorstellung

- Vorliegen einer entsprechenden Schulung für die beteiligten Personen (Ärzte, medizinisches Personal) in der Anwendung der telemedizinischen Methoden

- Durchführung unter Wahrung etablierter Qualitätsstandards, wie z.B. des Facharztstandards

- Wahrung von Standards zur Aufklärung, Dokumentation, Datenschutz und Datenspeicherung

- Einhaltung transparenter rechtlicher Rahmenbedingungen

- Wissenschaftliche Begleitung des Telemedizin-Projektes

Infobox 5 Aspekte, die auf einen sinnvollen Einsatz von telemedizinischen Methoden hinweisen können
Aspekte der Datenspeicherung

Aus berufsrechtlicher Sicht besteht die Verpflichtung zur Aufbewahrung der Untersuchungsdaten für mindestens 10 Jahre. Da zivilrechtliche Ansprüche in Deutschland erst nach 30 Jahren verjähren, wurde empfohlen, alle Behandlungsdaten für diese Dauer aufzubewahren [48]. Das aber würde nicht nur im telemedizinischen Kontext schon aufgrund von Kompatibilitätsproblemen im Rahmen der Weiterentwicklung von informationstechnologischen Standards eine Herausforderung darstellen. Das E-Health-Gesetz [34] führt deshalb das Interoperabilitätsverzeichnis vesta (https://www. vesta-gematik.de/) ein, um die im Gesundheitswesen verwendeten Standards transparent zu machen, die Finanzierung der neuen Anwendungen zu sichern und die Probleme für die Telemedizin zu vereinfachen und informationstechnologische Standards zu definieren.

\section{Aspekte des Schutzes von Privatdaten}

Das Strafgesetzbuch regelt in $§ 203$ StGB den Schutz von Privatdaten, die jemand in einer Eigenschaft als Arzt anvertraut und bekannt geworden sind. Derjenige, der unbefugt solche Geheimnisse offenbart, kann mit Freiheitsstrafe bis zu einem Jahr oder mit Geldstrafe bestraft werden. Auf EU-Ebene müssen zusätzlich die Europäische Grundrechte-Charta und ab dem 25.05.2018 die geltende EU-Datenschutzgrundverordnung (EU-DSGVO) beachtet werden. Die EU-DSGVO ist in diesem Zusammenhang von Bedeutung, obwohl sie inhaltlich für die telemedizinischen Netzwerke wenig ändert, da diese in der Regel einen benannten Datenschutzbeauftragten bereits vorhalten mussten. Aber nun erreichen die möglichen Bußgelder mit bis zu 10 Millionen Euro eine neue Dimension, die eine juristische Begleitung bei der Konzeption telemedizinscher Netzwerke sinnvoll erscheinen lassen.

\section{Fernbehandlungsverbot}

Traditionell war eine ausschließliche Behandlung von Patienten über Print- oder Kommunikationsmedien und nicht persönlich durch das sogenannte Fernbehandlungsverbot ausgeschlossen, verankert in $\S 7$ Absatz 4 der Muster-Berufsordnung (MBO) für Ärzte. Allerdings waren in den Erläuterungen der Bundesärztekammer zulässige Modelle für telemedizinische Verfahren näher definiert worden [49]. Diese umfassten 7 prinzipiell mögliche Szenarien der Fernbehandlung, bei denen nur der direkte Arzt-PatientenKontakt (Modell 7) eines für den Arzt „unbekannten“ Patienten als berufsrechtswidrig eingestuft wurde. „Unbekannt“ wurde in diesem Kontext wie folgt definiert: Ein Patient, bei dem der behandelnde Arzt keine eigene körperliche Untersuchung durchgeführt, keine Kenntnisse zu dessen wesentlichen Vorbefunden, keine medizinischen Informationen über den Krankheitsverlauf und keine Kenntnisse über das soziale Umfeld des Patienten hat.

Der 121. Ärztetag 2018 in Erfurt hat diese Regelung durch Änderung der Muster-Berufsordnung aufgehoben. Der entsprechende Absatz lautet nun wie in > Infobox 6 dargestellt.

Gemäß den Erläuterungen der Bundesärztekammer [50] soll so einerseits grundsätzlich die ärztliche Beratung und Behandlung im persönlichen Kontakt als Goldstandard etabliert bleiben, andererseits eine Unterstützung ärztlicher Tätigkeiten durch digitale Techniken gefördert werden. 
II. Pflichten gegenüber Patientinnen und Patienten

\section{$\S 7$ Behandlungsgrundsätze und Verhaltensregeln}

„(4) Ärztinnen und Ärzte beraten und behandeln Patientinnen und Patienten im persönlichen Kontakt. Sie können dabei Kommunikationsmedien unterstützend einsetzen. Eine ausschließliche Beratung oder Behandlung über Kommunikationsmedien ist im Einzelfall erlaubt, wenn dies ärztlich vertretbar ist und die erforderliche ärztliche Sorgfalt insbesondere durch die Art und Weise der Befunderhebung, Beratung, Behandlung sowie Dokumentation gewahrt wird und die Patientin oder der Patient auch über die Besonderheiten der ausschließlichen Beratung und Behandlung über Kommunikationsmedien aufgeklärt wird.“

- Infobox 6 Auszug aus der Muster-Berufsordnung der Ärzte [50]

Somit ist jetzt auch die telemedizinische Betreuung von Patienten, die sich erstmalig vorstellen, prinzipiell möglich, wenn die weiteren Anforderungen zur ärztlichen Sorgfalt und zur Aufklärung der Patienten über Vor- und Nachteile der telemedizinischen Betreuung erfüllt werden. Die Neuregelung löst auch teilweise den Gegensatz zwischen der MBO und der Berechtigung des Patienten zur freien Arztwahl bzw. der Möglichkeit, telemedizinische Beratung vom europäischen Ausland aus in Deutschland anzubieten. So erschließt diese Änderung der MBO den deutschen Gesundheitsmarkt für die ärztliche Beratung auch aus dem Ausland.

Allerdings stehen andere Regelungen dieser Öffnung im Bereich der Therapie entgegen:

So sieht das Sozialministerium Baden-Württemberg [51] die nach $\S 48$ Abs. 1 Satz 2 des Arzneimittelgesetzes (Abgabeverbot bei Fernverschreibungen) die Verordnung eines Arzneimittels ohne „offenkundigen“ Arzt-Patienten-Kontakt als grundsätzlich verboten an. Dieses Verbot könne nur ,in begründeten Ausnahmefällen“ umgangen werden. Diese Einschätzung wurde auch nach Diskussion im Landtag Baden-Württemberg nicht revidiert [52].

Die Bescheinigung einer Arbeitsunfähigkeit erfolgt nach $\S 31$ des Bundesmantelvertrags [53] in Verbindung mit der Arbeitsunfähigkeits-Richtlinie des Gemeinsamen Bundesausschusses (GBA). Diese fordert eine ärztliche Untersuchung als Grundlage der Einschätzung, ob und wie lange eine Arbeitsunfähigkeit besteht. In beiden Regelungen ist eine telemedizinische Untersuchung nicht erwähnt. Daher wurde dies als fortbestehende rechtliche Hürde für eine telemedizinische Behandlung gesehen [51]. Dem ist entgegen zu halten, dass auch eine tatsächlich durchgeführte körperliche Untersuchung im Bundesmantelvertrag nicht gefordert wird [54]. Der GBA hat in § 4 seiner Arbeitsunfähigkeits-Richtlinie explizit eine ärztliche Untersuchung in Satz 2 gefordert, jedoch findet sich auch hier kein Hinweis darauf, dass diese in persona durchzuführen sei.

Damit könnte die Änderung der Musterberufsordnung zu dem Analogschluss führen, dass bei zunehmend besserer Studienlage mit vergleichbarer Verlässlichkeit von telemedizinisch und im direkten Patientenkontakt erhobenen Untersuchungsbefunden auf individueller Basis und der Grundlage einer sorgfältigen Dokumen- tation auch die Art der Befunderhebung für die Bescheinigung der Arbeitsunfähigkeit irrelevant sein müsste. Dieser Einschätzung steht allerdings ein weiterer Beschluss des 121. Ärztetages 2018 [55] entgegen, nach dem die Ausstellung einer Arbeitsunfähigkeitsbescheinigung abgelehnt werden soll, wenn es im Rahmen einer ausschließlichen Fernbehandlung mit der betreffenden Person zu keinem persönlichen Arzt-Patienten-Kontakt kommt. Ferner gilt weiterhin der Sicherstellungsauftrag, der die Fernbehandlung im vertragsärztlichen Bereich ausschließlich durch Vertragsärztinnen und - ärzte vorsieht, um so eine Konkurrenz mit kapitalorientierten Gesellschaften zu vermeiden [56].

Hier werden erst die künftige Rechtsprechung und weitere, ggf. detaillierte Gesetzgebung helfen, die Sachlage besser einschätzen zu können, und so die Rechtssicherheit der handelnden Personen zu erhöhen.

\section{Weitere rechtliche Aspekte}

Im Falle der Fehlbehandlung auf telemedizinischem Wege stellen sich in Abhängigkeit der beteiligten Personen (z. B. direkter ArztPatienten-Kontakt, Tätigkeit als Tele-Konsiliar [57]) sowie der Vertragsgestaltung (z. B. Gesetzliche Krankenversicherung, Private Krankenversicherung, Vertragsgestaltung der Beteiligten zur Haftung im Rahmen des Projektes [57, 58], Art der Finanzierung) eine Vielzahl von Fragen, die bei Sitz des telemedizinischen Netzwerkes im Ausland sogar Fragen europäischen und internationalen Rechts berühren mögen. Sicherlich werden Art und Wirksamkeit der zusätzlich geforderten Aufklärung über die telemedizinische Natur der Behandlung und die Sicherstellung der Sorgfaltspflicht im Rahmen des Kontaktes mit Wahrung des Facharztstandards im Falle eines Rechtsstreites ausreichende Ansatzpunkte für anwaltliche Nachfragen ergeben. Die schon in der MBO geforderte Dokumentationspflicht kann positiv gesehen zur Klärung derartiger Sachfragen beitragen, mag aber aufgrund der fehlenden DokumentationsStandards selbst auch ein Ansatzpunkt sein, den strittig fehlerhaften Vorgang negativ zu beurteilen, wenn das Beibringen von digitalen Dokumenten (wie oben ausgeführt auch 10 Jahre nach erfolgter Behandlung) nicht in ausreichendem Maße gelingt.

\section{Zusammenfassende Bewertung der rechtlichen Situation}

Trotz der Öffnung in der MBO mit prinzipieller Möglichkeit zur Durchführung von telemedizinischen Methoden verbleiben somit zahlreiche interessante zivil-, straf- und berufsrechtliche Risiken, die eine besonders sorgfältige Planung und Umsetzung derartiger Arzt-Patienten-Kontakte erforderlich machen. Andererseits sollten die aufgeführten Aspekte nicht von der Entwicklung telemedizinscher Projekte abschrecken, da unter fachlicher Begleitung dank der Änderung der MBO sich die Rechtssicherheit für die Telemedizin deutlich erhöht hat und das eHealth-Gesetz [34] die Umsetzung einer Video-Sprechstunde explizit fördert.

\section{Medizinische und technische Grenzen der Telemedizin}

Die Notwendigkeit, gesundheitliche Probleme im Rahmen einer HNO-ärztlichen Vorstellung nicht nur festzustellen, sondern auch zu beheben, ist eine anerkannte Limitation. So wurde z. B. die mangelnde Entfernbarkeit von Ohrschmalz als Grenze der HNO-Telemedizin eingeschätzt [59]. 
Weiterhin wurde die Notwendigkeit durch Palpation zusätzliche Informationen zu erheben, als wichtig und besonders für die Untersuchung von Halsveränderungen limitierend eingeschätzt [59]. Um diese Probleme zu lösen, werden mittlerweile tragbare haptische Systeme für Hand bzw. Finger entwickelt, die dann den Palpationseindruck übertragen sollen, bzw. es wird Ultraschall verwendet [60,61].

Technische Barrieren bestehen weiterhin u. a. in der lückenhaften Abdeckung mit Breitbandnetzwerken [62] und den fehlenden informationstechnologischen Standards.

\section{Akzeptanz telemedizinischer Methoden und Geräte in der Bevölkerung}

Eine weitere Limitation ist die Empfänglichkeit der Betroffenen also HNO-Ärzten, Patienten und medizinischem Personal - für telemedizinische Methoden, die damit verbundenen technischen Geräte und Aufgaben.

Bereits 2009 konnte in einem deutschen Survey die technische Ausstattung zur digitalen Kommunikation zwischen Patienten und Ärzten der HNO-Heilkunde als grundsätzlich ausreichend weit verbreitet angesehen werden. So besaßen zu diesem Zeitpunkt mehr als $80 \%$ einen Computer mit Internetanschluss (jüngere Patienten bis zu einem Alter von 45 Jahren: über $90 \%$ ), sowie knapp $50 \%$ ein Handy mit Internetanschluss.

Die Befragung von 250 Patienten einer HNO-Universitätsklinik [63] wies mittels einer Teilnahmerate von $84,4 \%$ auf das hohe Interesse an Fragen zu Telemedizin hin. Bei 2003 auswertbaren Datensätzen zeigte sich eine sehr hohe Verbreitung technischer Geräte, die telemedizinisch genutzt werden können (Mobiltelefon: 94,6\%; Computer mit Internetzugang: 85, 1 \%) und im Vergleich zu 2009 eine deutliche Steigerung. Der Vergleich nach dem Vorstellungsgrund ergab dabei besonders hohe Verfügbarkeitsdaten von Smartphone (97,8\%) und Computer mit Internetzugang (97,8\%) für Patienten mit nasalen Beschwerden, gefolgt von Patienten mit Ohr(94,3\%; 86,4\%) und Hals-Symptomen (95,9\%; 74,5\%). Dabei wurden Alter, Beruf und Bildungsniveau als Einflussfaktoren für den Gebrauch von moderner Kommunikationstechnologie nachgewiesen.

Die Bereitschaft online mit dem behandelnden HNO-Arzt Kontakt aufzunehmen, besteht bei ca. der Hälfte der Patienten. So sagten 51,9\% der Teilnehmer die Bereitschaft zu, Medikationspläne digital vor einem Termin zu schicken und 52,3\% gaben an, Befunde nach einem Arzttermin gerne digital zu erhalten. Potenzial zur Verbesserung des Arzt-Patienten-Kontaktes sahen nur ca. ein Drittel der Patienten. Das ist aber auch methodisch begründbar, schließlich erfolgte die Befragung unter Patienten, die in der Lage waren, die HNO-Universitätsklinik persönlich aufzusuchen und damit nicht bei den primären Adressaten eines telemedizinischen Netzwerkes. Dabei scheinen Privatpatienten den neuen technologischen Möglichkeiten offener gegenüber zu stehen, besonders einer Erinnerungsfunktion für Termine (odds ratio (OR) 3,69, 95 \% Konfidenzintervall $(95 \% \mathrm{Cl}) 1,26-10,79)$ und dem online Transfer von medizinischen Informationen (OR 2,14, 95 \% Cl 0,94-4,82). Insofern scheint für den HNO-Bereich auf der Patientenseite eine ausreichend hohe Bereitschaft zu bestehen, telemedizinische Kontakte auszubauen.
HNO-Patienten nutzen das Internet auch zur Vorbereitung eines Arztbesuches: So gaben in einer Untersuchung aus dem Jahre 2014 ca. 75 \% an, im Internet in Bezug auf Ihre Erkrankung und $50 \%$ in Bezug auf den zu konsultierenden HNO-Arzt recherchiert zu haben $(N=79)[64]$.

\section{Akzeptanz telemedizinischer Methoden in der Ärzteschaft}

In den Anfängen telemedizinischer Netzwerke war in der Ärzteschaft die Sorge groß, dass durch substituierende Prozesse auch HNO-spezifische Tätigkeiten von anderem medizinischen Personal übernommen werden könnten. So wurden die Versorgungsassistentin in der Hausarztpraxis VERAH [65-70] oder AGNES (arztentlastende, gemeindenahe, E-Health-gestützte, systemische Intervention) [65, $71-$ 73] und ähnliche Konzepte als Modelle angesehen, die bei Verknüpfung mit einem telemedizinischen Netzwerk den Tätigkeitsbereich einschränken und in den Bereich der Allgemeinmedizin verlagern könnten. Dank der klaren Stellungnahme der Bundesärztekammer gegen substituierende Prozesse [18] einerseits, aber auch den zunehmenden Berichten über den Aufgabenbereich von VERAH und AGNES konnten hier Hemmnisse abgebaut werden.

Da VERAH sich primär um Aufgaben aus dem Bereich Organisatorische Leistungen, Betreuungs- und Unterstützungsleistungen mit Beratungen zu Impfschutz, zur Inanspruchnahme von Vorsorgeleistungen, Arztkontrollen und Einnahmetreue von Tabletten kümmert, sind hier positive Effekte auch für Termine beim HNO-Arzt vorstellbar $[66,67,70]$. Der Einsatz zur Entlastung von Allgemeinmedizinern wurde von den ärztlichen Kollegen, den betreuten Patienten und den Versorgungsassistenten positiv bewertet. Ein Projekt mit direkter Relevanz für die HNO-Heilkunde konnte im Rahmen der systematischen Literatursuche nicht identifiziert werden.

AGNES dagegen wurde im ländlichen Raum von MecklenburgVorpommern auch in Verbindung mit einem telemedizinischen Netzwerk eingesetzt. Die telemedizinische Methodik trug zur Untersuchung der Medikamenten-Interaktion und der Messung des Augen-Innendrucks bei [72, 73]. Dies bestätigt prinzipiell den Einsatz telemedizinischer Techniken auch in einem substituierenden Konzept. Jedoch lassen fehlende aktuelle Berichte der letzten 9 Jahre vermuten, dass derzeit dieser Weg wohl nicht weiterverfolgt wird.

Die Bereitschaft, in einem telemedizinischen Netzwerk zu arbeiten, hängt für HNO-Ärzte weiterhin von technischen und berufspolitischen Faktoren [74, 75] ab.

Zur technischen Bereitschaft erfolgte eine Umfrage unter 334 HNO-Ärzten, die in Privatpraxen tätig waren, mittels standardisiertem Fragebogen [76]. Bei nur 47 \% vollständig auswertbaren Fragebögen zeigten sich die Praxen mit knapp $90 \%$ der elektronischen Gesundheitsakte technisch gut vorbereitet. Allerdings erfolgte die Kommunikation zu Partnern außerhalb der Praxis in mehr als $60 \%$ der Fälle analog, z. B. per FAX. Verbesserungspotenzial und Anwendungsmöglichkeiten wurden primär im Bereich der Kommunikation mit anderen Gesundheitseinrichtungen (Hausärzten, Krankenhäusern) sowie der Terminvergabe und -erinnerung gesehen.

Die möglicherweise berufspolitisch motivierte Zurückhaltung wurde schon 2013 im Hinblick auf eine Skepsis von 55\% der HNOÄrzte diskutiert $[74,75]$. Dabei erfolgte die Befragung in einem 
Netzwerk [75], das in einem urbanen Umfeld die telemedizinische Zusammenarbeit von Allgemeinmedizinern mit HNO-Ärzten, also als fachfremdes Telekonsil gemäß \ Infobox 2, anbot. Damit waren die Rahmenbedingungen - eine Pilotstudie, geringe ersichtliche Vorteile bei einer Bewertung anhand von Faktoren der $>$ Infobox $\mathbf{5}$ und ein relativ frühes Entwicklungsstadium der HNO-Telemedizin - zu ungünstig, um ein besseres Ergebnis zu erreichen.

Bekanntlich bezieht sich die Zulassung eines Arztes und seine Abrechnung mit der Krankenkasse eines Patienten jeweils auf einen bestimmten Arbeits- bzw. Wohnort. Deshalb sind Hemmnisse durch Landesgrenzen, wie im Bereich Benelux/Frankreich/Deutschland, trotz guter Kommunikationsmöglichkeiten auch eine Limitation zum Angebot telemedizinischer Leistungen. In den USA ist diese Barriere noch ausgeprägter, da die Zulassung des Arztes dem jeweiligen Bundesstaat obliegt und so trotz gleicher Sprache und Staatsangehörigkeit ggf. eine telemedizinische Behandlung nicht finanziert wird [77].

\section{Krankheitsbilder und Indikationen}

Einen Überblick über die vielfältigen Telemedizin-Projekte in Deutschland bot das Deutsche Telemedizin-Portal, das im Rahmen der eHealth-Initiative des Bundesministeriums für Gesundheit unter enger Mitarbeit der Bundesärztekammer aufgebaut wurde. Interessierten Ärzten und Patienten oder Initiatoren von Telemedizin-Projekten stellte das frei zugängliche Portal zahlreiche Informationen bereit. Mit Umzug und Neu-Strukturierung der aufgeführten Projekte ab September 2018 unter https://www.informationsportal.vesta-gematik.de ist hier auch für das Fachgebiet der Hals-Nasen-Ohrenheilkunde ein deutlicher Datenverlust zu konstatieren. Die Registrierung der Projekte ist weiterhin freiwillig. Sie geben aber aufgrund von Interessenskonflikten um Drittmittel, geistiges Eigentum und Informationstechnologischen-(IT)-Strukturen der Betreiber von Telemedizin- Netzwerken nicht mehr einen vollständigen Überblick über die aktuellen Projekte.

Um diese Übersicht über die Eignung telemedizinscher Verfahren zur Beurteilung häufiger Krankheitsbilder der HNO-Heilkunde zu gewinnen, steht u. a. eine retrospektive Untersuchung zur Verfügung [38], die $62 \%$ aller HNO-ärztlichen ambulanten Vorstellungen als prinzipiell einer telemedizinischen Vorstellung zugänglich beurteilt. Erkrankungen von Innen- und Mittelohr wurden hier eher für die Telemedizin geeignet eingestuft als Erkrankungen von Larynx oder äußerem Ohr [38]. Dagegen wurden Fälle, in denen eine apparative Untersuchung für eine Diagnosestellung benötigt wird, als ungeeignet für die Telemedizin klassifiziert. Als Ergebnis scheint, nicht zuletzt aufgrund zwischenzeitlicher Entwicklungen auf dem Gebiet der Tele-Audiometrie, aus der Sicht des niedergelassenen HNO-Arztes ein relevanter Patientenanteil auch telemedizinisch betreubar zu sein. Um zu klären, welche Patientengruppen dabei in besonderem Maße geeignet sind, erfolgte die Evaluation verschiedener Patientenkohorten.

\section{Gemischte Patientenkohorten}

Postoperative Vorstellungen

Telemedizinische Visiten wurden v.a. (zu 70\%) postoperativ nach Kopf-Halschirurgie in einer 250 Patienten umfassenden Kohorte durchgeführt [78]. Etwa ein Drittel der Patienten zeigte einen Status nach Operationen der Mundhöhle und des Oropharynx (wie Sialendoskopie oder Tonsillektomie), ein weiteres Drittel nach größeren halschirurgischen Eingriffen, wie Operationen der Schilddrüse, der Glandula parotis oder einer Neck dissection. 30\% aller Visiten wurden als klinische Nachkontrollen zur Besprechung von Labor- oder histologischen Befunden klassifiziert. Für diese Kohorte dauerten die Visiten im Durchschnitt $11 \mathrm{~min}$. (0-47 min.) bei einer Wartezeit für die Patienten von 10 min. Die Patienten waren zu $95 \%$ mit der Vorstellung zufrieden und gaben an, zu $88 \%$ eine Telekonsultation erneut nutzen zu wollen. Kritikpunkte betrafen v. a. technische Probleme inkl. Verbindungsprobleme. $46 \%$ der Patienten konnten so mehr als 3 Stunden Reisezeit einsparen, weitere $40 \%$ gaben die Einsparung von 1-3 Stunden Reisezeit an.

Eine weitere interessante Untersuchung evaluierte die Qualität einer photographischen Wunddokumentation von 37 Frauen nach dermatologischen Eingriffen [79]. So konnte eine Spezifität von mehr als $90 \%$ nachgewiesen werden. Verschiedene Beurteilungen der Wunden zeigten eine sehr hohe Übereinstimmung (Intraclass correlation coefficient [ICC] R=0,806 (95\% CI 0,694, 0,881)) bei Verwendung der ASPSIS Skala zur Beurteilung postoperativer Wunden [80], was auf eine gute Kongruenz der klinischen Einschätzung hindeutet. Lediglich die Feststellung eines lokalen Erythems war hier im Vergleich schwieriger [79]. Dies mag einen Hinweis auf eine technische Abhängigkeit, z. B. auf die Belichtungssituation, geben. Neben anderen wissenschaftlichen Studien mögen die hier angeführten Daten nachvollziehbar machen, weshalb postoperative Vorstellungen eine anerkannte Indikation zur Videosprechstunde darstellen (vgl. > Infobox 4).

\section{Fachgleiche Netzwerke}

Eine relativ alte Untersuchung verglich die Übereinstimmung der gestellten Diagnosen bei Untersuchung durch einen HNO-Assistenzarzt unter Verwendung einer Live-Videokonferenz bzw. einer Speicherung der Untersuchungsergebnisse und zeitversetzter Befundung mit dem Goldstandard - der Diagnose vor Ort durch einen Facharzt [81]. Demnach stimmten HNO-Assistenzarzt und Facharzt vor Ort in 92\% (36 von 39) der Fälle einer heterogenen Diagnoseliste überein. Der Tele-Konsiliar kam mittels Videokonferenz in $85 \%$ zu dem gleichen Ergebnis. Bei zeitversetzter Versendung sank die Übereinstimmungsrate auf $64 \%$ ab. Diese Untersuchung weist bei einer nur begrenzten Fallzahl einerseits auf das Risiko einer nicht dem Goldstandard entsprechenden Diagnose bei Verwendung telemedizinscher Methoden in 7-28\% der Fälle hin. Zusätzlich illustriert sie eindrücklich den Einfluss einer Live-Videokonferenz zur Verbesserung der korrekten Diagnosestellung in einer relevanten Größenordnung von 20\%. Eigene Untersuchungen primär laryngologischer Befunde von 250 Patienten bestätigen die Größenordnung der Abweichungen [82]: So zeigte sich eine Übereinstimmung der Diagnosen zwischen persönlicher Untersuchung durch einen HNO-Facharzt und zeitversetzter, verblindeter Beurteilung eines Tele-Konsiliars in $83,1 \%$ bei hoher Übereinstimmungsrate für daraus abgeleitete Therapievorschläge von 94,1\%. Bei den abweichenden Beurteilungen wurden im Rahmen der Zweitbeurteilung zweimal unnötige Operationen (,Übertherapie') angeraten und dreimal eine Operationsindikation nicht gestellt. 
Eine prospektive Untersuchung von 62 Patienten mit Halsschmerzen [83] weist auf mittlere Übereinstimmungen bei telemedizinische Befundung einerseits und persönlicher Untersuchung andererseits für oberflächliche Lymphknotenveränderungen sowie Gaumenrötung hin. Der Bereich des hinteren Pharynx, submandibuläre Lymphknoten und die Größe der Tonsilla palatina wurden nicht ausreichend gut beurteilt. Damit scheinen diese anatomischen Regionen mit telemedizinischen Methoden derzeit nicht hinreichend sicher beurteilbar zu sein.

Zusammenfassend betonen die Daten aus rein HNO-ärztlichen Telemedizin-Netzwerken die relativ hohe Zuverlässigkeit der Diagnosestellung, die sich apparativ durch Verwendung einer Videokonferenz optimieren lässt. Gleichzeitig bestehen deutliche Qualitätsunterscheide bei der telemedizinischen Beurteilung der anatomischen Untereinheiten unseres Fachgebietes, die Anlass zur Weiterentwicklung sein müssen.

\section{Fachfremde Netzwerke}

Besonders interessant für eine flächendeckende Verbesserung des Zugangs zu HNO-ärztlichem Wissen ist die telemedizinische Zusammenarbeit mit Ärzten anderer Fachrichtungen (fachfremde Telekonsile). So wurden prospektiv $50 \%$ der HNO-ärztlichen Vorstellungen einer heterogenen Kohorte $(\mathrm{N}=48)$ als vermeidbar durch ein Tele-HNO-Konsil eingestuft [59], wenn zeitlich vorgeschaltet eine Untersuchung durch einen Allgemeinarzt mit Live-Unterstützung durch einen HNO-Arzt stattfand. Auch die durchschnittliche Übereinstimmung des Ergebnisses von Tele-Konsil und persönlichem Kontakt erscheint in der Gesamtgruppe mit 79.2\% hoch, wobei sie mit nur noch $60 \%$ in der Untergruppe von Patienten mit Hals-, Pharynx- oder Larynxproblemen deutliche Schwächen offenbart. Leider werden die konkreten Diagnosen und Indikationen auch im Volltext nicht aufgeführt. Zu kritisieren ist weiterhin die Verwendung von Skype zur Übertragung, da diese - zumindest aus deutscher Sicht - den Datenschutzanforderungen für eine Arzt-zuArzt- Kommunikation unter Preisgabe sensibler Daten nicht ausreichend Rechnung trägt [59].

Ein ältere Untersuchung weist in diesem Kontext auf die Lernkurve hin, die die fachfremden Untersucher durchlaufen: Bei endoskopischer Untersuchung von 42 Patienten (55 Diagnosen) führte die Lernkurve in der Untersuchungstechnik des Allgemeinmediziners vor Ort bei 8 der ersten 20 Patienten zu fehlerhaften Diagnosen [84]. So wurde bei 3 Patienten die falsche Diagnose gestellt und in weiteren 5 Fällen eine Diagnose nicht gestellt. Das Diagnosespektrum war heterogen mit Beschwerden im Bereich der Ohren inkl. Neurootologie, der Nase sowie Halsschmerzen, jedoch ohne laryngologische Fragestellung.

Damit illustrieren diese Daten eindrücklich die Wichtigkeit einer Schulung in HNO-ärztlicher Untersuchungstechnik und auch die Gefahr einer Fehldiagnose bei rein telemedizinischer, fachfremder Beurteilung besonders im Bereich von Pharynx, Larynx und Hals.

\section{Daten aus homogenen Patientenkohorten}

In den verschiedenen anatomischen Bereichen des HNO-Fachgebietes variiert sowohl die Zuverlässigkeit einer telemedizinischen Beurteilung stark, als auch die Studienlage unter Beachtung der Anwendungsbereich Telediagnostik, Telekonsil, Telekonsultation,
Telemonitoring und Teletherapie. Daher sollen im Folgenden die Daten homogener Kohorten vorgestellt werden:

\section{Otologie}

Der klinische Goldstandard in Deutschland zur Beurteilung von Gehörgang und Trommelfell besteht im Einsatz der Ohrmikroskopie. Alternativ wird v. a. von Kinder- und Allgemeinmedizinern das Otoskop verwendet. In telemedizinischen Studien wird regelmäßig ein Video-Otoskop eingesetzt [14]. Um die technische Gleichwertigkeit zum ohrmikroskopischen Befund zu prüfen, erfolgte eine prospektive Untersuchung. Dabei beurteilten 12 Neurootologen video-otoskopische Aufnahmen, die anhand des Goldstandard eines Ohrmikrokops mit audiologischen Untersuchungen von 7 Diagnosekategorien zugeordnet worden waren [85]. Die Übereinstimmung für normale Trommelfelle lag bei nur $72 \%$. Die Übereinstimmung bei verschiedenen Pathologien variierten von 48,6-100\% [13-15, 85-88]. Bei simultanem, verblindetem Einsatz eines HNOFacharztes vor Ort und als Telekonsil konnte bei 21 Patienten eine Patientenzufriedenheit von $96 \%$ erreicht werden [89]. Ein Zeitgewinn für die Dauer der Konsultation konnte nicht nachgewiesen werden. Die Übereinstimmungsrate für die Diagnosen betrug $95 \%$. Technisch konnte bei allen Fällen ein ausreichend hochwertiges Bildmaterial übermittelt werden, allerdings versagte in einem Fall die Tonübertragung.

Eine weitere Studie berichtete von einer technisch ausreichenden Bildqualität bei $87 \%$ der Patienten in einem fachfremden Netzwerk (Voruntersuchung durch einen Allgemeinmediziner). Eine Diagnosestellung misslang bei $18 \%$, was zu einer konventionellen Nachuntersuchung führte. Die Übereinstimmung von konventioneller Ohrmikroskopie mit Video-Otoskopie zwischen Allgemeinmedizinern und HNO-Ärzten erschien insgesamt gut, so dass für den Einsatz in sehr ländlichen Gebieten dieses Vorgehen empfohlen wurde [90].

In Indien konnten so durch Training von Gesundheitsmitarbeitern mittels Video-Otoskopie (Smartphone-basiert) innerhalb 6 Monaten 3000 Patienten auf das Vorliegen von Ohrerkrankungen gescreent werden [91]. Es führte in $54 \%(N=1619)$ zur Empfehlung einer HNO-ärztlichen Vorstellung, über 215 Patienten wurde direkt berichtet. Diese erhielten in $50 \%$ der Fälle eine Operationsempfehlung. Damit erscheint in stark unterversorgten, ländlichen Regionen Indiens die Video-Otoskopie eine wertvolle Technik zu sein, um eine offensichtliche Unterversorgung zu bessern.

Damit dürfte auf dem Boden der derzeitigen Studienlage eine video-otoskopische Beurteilung in einem telemedizinischen Netzwerk ausreichend verlässlich sein. Entsprechend erfolgt der Einsatz u. a. als Teil einer telemedizinischen therapeutischen Intervention bei schwerhörigen Kindern in Australien [92].

\section{Tele-Audiometrische Untersuchungen}

Speziell in Entwicklungsländern wurden verschiedene audiometrische Untersuchungen erprobt, um telemedizinisch die Erkennung von Schwerhörigkeit zu bessern [43, 93-102]. Eine vergleichende Untersuchung wies bei Verwendung eines Computer-gesteuerten Tonaudiometers im Vergleich zu einer doppelt-blind durchgeführten, konventionellen Audiometrie eine Genauigkeit von 1,3 dB für Werte der Luftleitung und 1,2 dB für Messwerte der Knochenleitung nach [103, 104]. Die hohe Genauigkeit bestätigte eine ame- 
rikanisch-afrikanischen Studie [105], in der unterstützt durch ein Videokonferenz-System 30 Erwachsene untersucht worden sind. Ein Messunterscheid von mehr als $10 \mathrm{~dB}$ wurde nur bei $4 \%$ der Teilnehmer festgestellt. Allerdings dauerte die telemedizinische Messung etwas länger (10,4 vs. 8,2 min). Weitergehende Untersuchungen bestätigten die hohe Verlässlichkeit auch für die Durchführung von Sprachaudiogrammen [106]. In Untersuchungen aus Tadschikistan bzw. Polen wurde zur Früherkennung von Hörstörungen die Kombination von audiometrischer Untersuchung und 2 standardisierten Fragebögen verwendet, um kindliche Schwerhörigkeiten sicher zu erkennen [99]. Damit kann eine Tonaudiometrie telemedizinisch zuverlässig durchgeführt werden.

Zur telemedizinischen und möglichst automatischen Feststellung einer Schwerhörigkeit wurden verschiedene Tests entwickelt, die auch am Telefon bzw. im Internet durchführbar sind [107]. So wird der Speech-in-Noise-Test von Smits [101] u. a. in epidemiologischen Untersuchungen der UK Biobank durchgeführt und in einer auf Deutsch übersetzten Variante auch im Hörmodul der Nationalen Kohorte [108, 109].

Eine vergleichende Studie zur telemedizinischen oder konventionellen Unterstützung von Familien mit schwerhörigen bzw. tauben Kindern [110] zeigte, dass bei höherer Bewertung auch der telemedizinischen Visiten durch die betroffenen Familien sich die expressive Sprachentwicklung der Kinder verbesserte und gleichzeitig Kosten eingespart wurden. So konnten durch eine Anwendung tele-audiometrischer Verfahren Wartezeiten erheblich reduziert werden (von 73 auf 29 Tage) [111]. Die Kombination aus Video-Otoskopie und tele-audiometrischen Verfahren wurde in Australien als besonders effektiv eingeschätzt, um die medizinische Versorgung von Kindern [112] auch der Aborigines zu verbessern [113-116].

\section{Cochlear Implantation}

In Deutschland ist audiologische Expertise ebenfalls knapp. Von Interesse ist daher auch aus hiesiger Perspektive, ob Krankenhäuser bei Implantation von Cochlear Implantaten $(\mathrm{Cl})$ wirklich einen Audiologen vor Ort vorhalten müssen, oder ob dieser im Bedarfsfalle auch mittels Telemedizin on-demand zugeschaltet werden kann. Diese Fragestellung untersuchte eine Studie aus St. Peterburg, die technisch vergleichbare Ergebnisse anhand von Messungen direkt intraoperativ nach 50 Cochlear Implantationen bescheinigte. Bei Messungen an Patienten sowohl im Kinder- wie auch Erwachsenenalter (0,7-48 Jahre) zeigten sich technisch die Auslösung von Stapediusreflexen nur in einem von 6 Kanälen unterschiedlich, bei vergleichbaren Ergebnissen für Impedanz und Field Telemetry (IFT). Tele-audiologische Messungen sind technisch 8,6 min schneller bei gleicher Qualität ( $N=50$; [117]) ableitbar. Dies kann eine Strategie sein bei Verknappung der audiologischen Expertise bzw. zunehmendem Kostendruck. So gaben die Autoren an, aufgrund dieser Daten audiologische Messung nur noch telemedizinisch an ihrem Krankenhaus durchzuführen. Das prinzipielle Einsparpotenzial bei telemedizinischem Einsatz des Audiologen wurde auch von einer kleinen Fallserie mit 8 Messungen unterstützt [118].

Die Nachsorge nach Cochlear Implantation wurde ebenfalls als „Remote Fitting“ („Entferntes Anpassen“) entwickelt, in dem aufbauend auf einer Hörgeräte-Entwicklung die Möglichkeit geschaffen wurde, die technischen Einstellungen des $\mathrm{Cl}$ in direktem Kontakt mit dem Patienten zu optimieren [119]. So kann der Sprachprozessor verändert und die technische Spezifikation kontrolliert werden. Dabei wurde die enorme Anfangsschwierigkeit bei der Einarbeitung der dezentralen Partner im Netzwerk bereits eingeräumt. Dieses preisgekrönte Netzwerk umfasst derzeit 21 dezentrale Zentren, die von 500 Patienten genutzt werden, um so Wege zum Zentrum einzusparen [120].

Telemedizinische Techniken wie das „Remote Fitting“ sind derzeit weder im Weißbuch „Cochlea-Implantat(CI)-Versorgung in Deutschland “ [123], noch in der sich in Überarbeitung befindenden S2k Leitlinie erwähnt [122]. Das Weißbuch spricht sich vielmehr für den Fall einer audiologischen Nachkontrolle dafür aus, diese „primär an der $\mathrm{Cl}$-versorgenden Einrichtung durchzuführen“ “ 123].

\section{Tinnitus}

Aufgrund des hohen Gesprächsanteiles im Rahmen einer TinnitusBehandlung in den Bereichen Diagnostik, Beratung und Therapie [124] erschien es logisch, auch bei dieser Indikation telemedizinische Verfahren zu erproben. Die mangelnde Verfügbarkeit qualifizierter Therapeuten für eine Verhaltenstherapie von Tinnitus, gerade in ländlichen Regionen, macht darüber hinaus eine Erprobung sinnvoll.

Als Screening-Instrumente für die Tinnitus-Patienten sind prinzipiell die oben bereits aufgeführten audiologischen Verfahren zugänglich. Fragebögen zur Diagnostik von Tinnitus [125] und den wichtigen Einflussfaktoren Depression und Angst [126] sind auch online verfügbar und validiert.

Zur Tinnitus-Therapie via Internet bzw. Email existiert eine ganze Reihe von Studien:

Randomisiert wurden 117 Patienten mit einer Tinnitusdauer von mehr als 6 Monaten durch ein Selbsthilfe-Manual unter E-MailKontakt versorgt. Bei einer relevanten Drop-out-Rate von mehr als der Hälfte der randomisiert eingeschlossenen Patienten zeigte sich eine signifikante Abnahme der Tinnitus-assoziierten Beschwerden.

So wurde ein progressives Tinnitus Management entwickelt, um bei Patienten mit Tinnitus, teilweise auf Grund von Hirnschäden, den Zustand mit Hilfe telefonischer Beratung durch einen Audiologen und einen Psychologen zu bessern [127]. In der nicht-randomisierten Beobachtungsstudie konnte eine Besserung der Beschwerden, gemessen mit dem Tinnitus Handicap Survey 12 und 24 Wochen nach Therapiestart berichtet werden, die jedoch nicht signifikant war. Da technisch keine Probleme beobachtet wurden und die Gruppengrößen relativ klein waren ( $N=12 / 15 / 9)$, wurde die fehlende Signifikanz zum Anlass genommen, eine randomisierte Studie unter verbesserten Bedingungen zu beginnen. Verbesserungspotenzial sah man in der Modifikation der verwendeten Informationsmaterialen und beim Einsatz einer Videokonferenz.

Für Tinnitus-Patienten wurde der Einsatz von Telemedizin zur Herstellung einer Verbindung mit anderen Betroffenen ebenfalls als hilfreich eingestuft [124]. Ziel ist hier, Einsamkeit und Rückzugstendenz durch den Tinnitus zu vermeiden [128]. Eine vergleichende Untersuchung zur Wertigkeit eines online-Forums im Vergleich zu einer Internet-basierten Verhaltenstherapie [129] zeigte die Internet-basierte Verhaltenstherapie eine signifikante Überlegenheit auf die subjektive Symptomatik, gemessen mit dem Tinnitus Handicap Inventar. Dies bestätigten frühere Studien [125, 130, 131], 
die der Therapie auch eine Nachhaltigkeit von mehr als 3 Monaten bescheinigen konnten. Dabei scheint die Internet-basierte Therapie einer konventionellen Gruppentherapie gegenüber überlegen zu sein [132]. Aktuelle Studien [133-138] verfolgen diesen Ansatz weiter und weisen darauf hin, dass Tinnitus für telemedizinische Ansätze ein geeignetes Krankheitsbild zu sein scheint.

\section{Neurootologie}

Im Bereich der Neurootologie wurde anhand eines Fallberichtes eines Patienten mit gutartigem Lagerungsschwindel die prinzipielle Umsetzbarkeit eines Telekonsils mittels Videokonferenzsystem berichtet [139].

Mutmaßlich aufgrund der speziellen Ausrüstung fehlen aber Studien, die Netzwerke, wie vom Deutschen Schwindel- und Gleichgewichtszentrum der LMU München angekündigt [140], auch telemedizinisch einsetzen, um die Wertigkeit von so eingeholten Zweitmeinungen zu erheben.

In einer Studie aus dem Bereich der Teletherapie wurde die Stand- und Gangunsicherheit von Patienten mit Multipler Sklerose $[141,142]$ bei konventioneller Physiotherapie $(N=25 ; 2 \times 40$ min pro Woche) verglichen mit einer Telerehabilitation, bestehend aus Übungen mit einer Xbox $360^{\circledR}$ Konsole unter Monitoring mittels Videokonferenz ( $\mathrm{N}=25 ; 4 \times 20 \mathrm{~min}$. / Woche). Nach 10 Wochen Therapie zeigten sich in beiden Gruppen signifikante Verbesserungen, gemessen mittels Posturographie [142], jedoch ausgeprägter in der teletherapeutischen Gruppe. Auch der Tinetti-Test zur Erkennung des Sturzrisikos zeigte sich in der teletherapeutischen Gruppe signifikant verbessert [141]. Aufgrund der verschiedenen intensiven Übungszeiten in beiden Gruppen sollte die Studie nicht als Nachweis einer Überlegenheit der Teletherapie missverstanden werden, sondern eher als Hinweis, dass auch auf diesem Wege einer Besserung der Stand- und Gangsicherheit erreicht werden kann. Inwieweit dabei tatsächlich die Verwendung der Videokonferenz in dem gewählten Maße nötig ist und ob nicht eine wiederholte Kontrolle zur Aufrechterhaltung der Compliance ausreichen würde, müssen zukünftige Studien klären.

Trotz der Attraktivität, bei gangunsicheren Patienten unnötige Wege zu vermeiden, liegen bislang noch recht wenige Studien zu telemedizinischen Verfahren vor. Ein Grund dafür mag in den bislang begrenzten therapeutischen Möglichkeiten und der mangelnden intersektoralen Zusammenarbeit von Allgemeinmediziner und HNO-Arzt liegen. Schwindel stellt aufgrund seiner enormen Prävalenz ein wichtiges Tätigkeitsfeld auch des Allgemeinmediziners dar, der die fachlichen Angebote der Hals-Nasen-Ohrenheilkunde nur zurückhaltend annimmt.

\section{Phoniatrie}

\section{Diagnostik und Therapie von Artikulationsstörungen}

Die telemedizinische Diagnostik von Sprachstörungen im Kindesund Jugendalter wurde im Rahmen der Diagnostik von Sprech- und Sprachstörung vergleichend untersucht [143]. Dabei konnten in einem technisch einfachen Aufbau mittels Mikrophon Artikulationsstörungen zuverlässig in Art und Ausmaß festgestellt werden. Eine unterstützende Videokonferenzanlage wurde gewünscht, um bei der Behandlung zusätzlich auch die nonverbale Ebene (durch Lächeln, Handbewegungen) einbeziehen zu können. Dabei wird empfohlen, dass an der dezentralen Untersuchungsstelle eine un- terstützenden Person, auch für die Behebung ggf. auftretender technischer Probleme, vorhanden ist. Gemäß einer Umfrage in Indien werden dort, aufbauend auf diesen Erfahrungen, derzeit ca. $12 \%$ der Sprachtherapien und -diagnostik auf telemedizinischem Wege durchgeführt. Ebenso wird aus ländlichen Gebieten in den USA [144] eine telemedizinische Umsetzung berichtet.

\section{Diagnostik und Therapie von Redeflussstörungen}

Telemedizinische therapeutische Interventionen wurden v. a. systematisch bei Redeflussstörungen wie dem Stottern [145] eingesetzt und wissenschaftlich begleitet. So konnte zunächst in einer 6 Kinder umfassenden Pilotstudie das Ausmaß des Stotterns auch telemedizinisch gebessert werden (von 13-36\% auf 2-26\%) mit fortbestehendem Effekt auf 6 Monate nach Therapieende.

Bei Redeflussstörungen wurde im Rahmen einer Pilotstudie bei Erwachsenen ( $N=16)$ Stammeln per E-Mail therapiert [146]. Dabei wurde die Kommunikation verwendet, um Therapieziele wie Persönlichkeitsentwicklung, Optimierung der Therapeuten-PatientenBeziehung und klinische Entscheidungsfindung zu verbessern.

In einer prospektiven, randomisierten Studie [147] wurde bei Erwachsenen ( $\mathrm{N}=40)$ die Therapie beim Stottern gemäß des Camperdown Programms telemedizinisch mit der Therapie im persönlichen Kontakt verglichen. Das Camperdown Programm ist ein in Australien entwickelter Therapieansatz zur Heilung des Stotterns $[148,149]$, der einen Schwerpunkt auf die Nachahmung der Sprechflüssigkeit setzt und insofern verhaltenstherapeutischen Prinzipien folgt. Es zeigte sich auch 9 Monate nach Therapieende kein signifikanter Unterschied zwischen den beiden Gruppen. Das deuteten die Autoren als Zeichen einer gleich hohen Therapieeffektivität bei sogar geringerer Kontaktzeit der auf telemedizinischem Wege behandelten Patienten. Diese vergleichenden Daten werden unterstützt von einer Phase-I-Studie ( $\mathrm{N}=10$; Erwachsene), die die Effektivität eines rein telemedizinischen Camperdown Programmes mit einer Reduktion des Stotterns unmittelbar nach Therapieende um $84 \%$ und 6 Monate später um 72 \% angab [150]. Aufgrund dieser positiven Ergebnisse schlossen sich weitere Untersuchungen zur Therapie von Jugendlichen an, wobei eine Webcam-basierte Therapie in der heimatlichen Umgebung verwendet wurde [151]. Dabei ergab eine Phase-I-Studie an 3 Jugendlichen eine Reduktion des Stotterns um $83 \%$ zum Therapieende bzw. um 93 \% bis 6 Monate danach. Die Ergebnisse waren damit erneut vergleichbar zu denen aus einer Phase-1-Studie der gleichen Altersgruppe bei persönlichem Patientenkontakt [152]. Nachteilig wurde allerdings bemerkt, dass in der Webbasierten Therapie Jugendliche weiterhin eine Tendenz zeigten, Sprachsituationen zu vermeiden [151]. Trotz Bestätigung der Effizienz in einer Phase-2-Studie von 14 Jugendlichen wiederholte sich hier das Problem, dass bei der Hälfte der Teilnehmer das Vermeidungsverhalten gegenüber Sprachsituationen sich nicht besserte [153]. Zusätzlich wurde das Studiendesign kritisiert [154], speziell die technische Erhebung der Nachhaltigkeit der Therapie. Deshalb sind trotz der prinzipiell hohen Studienqualität und-anzahl auch in diesem Bereich zusätzliche Studien zur Nachhaltigkeit und Wirksamkeit telemedizinischer Maßnahmen zu fordern. Bis diese vorliegen wäre es denkbar, durch zeitliche Vorschaltung des teletherapeutischen Ansatzes die Ausgangssituation für eine persönliche Situation zu verbessern, die Patientenbelastung durch Reise- 
belastung und -kosten zu mindern und so die Therapieakzeptanz zu steigern.

Die Amerikanische Speech-Language-Hearing Association (ASHA) beurteilte daher bereits 2005 eine telemedizinische Therapie als geeignete Behandlungsform bei Sprachstörungen, um Barrieren wie räumliche Distanz, mangelnde Verfügbarkeit von Spezialisten und Immobilität der Patienten zu überwinden und so die Versorgungssituation zu verbessern (nach [92]).

\section{Dysphagie-Behandlung}

Aufbauend auf existierende Strukturen im Bereich der Sprachtherapie werden mittlerweile auch Schlucktherapien telemedizinisch durchgeführt. Nach ersten Untersuchungen in Australien mit positiven Erfahrungen bei Anbindung eines regionalen Krankenhauses an ein Zentrum [155] und Behandlung von Kopf-Hals-TumorPatienten verbreitete sich dieser Therapieansatz. Die nötige Zuverlässigkeit in der Beurteilung des Schluckaktes liegt bei mittleren Übereinstimmungsraten (kappa $=0,636$ ) für die Penetrations-Aspirationsraten zwischen Untersucher vor Ort und Tele-Konsiliar (durchschnittliche absolute Differenz von 1,1 Punkten auf einer 8 -stufigen Skala) [156]. Untersucht wurden dabei 32 Patienten mit Schluckstörungen neurologischer Genese oder nach Kopf-HalsTumor. Die Übereinstimmung für Behandlungsempfehlungen schwankte zwischen exzellenten $100 \%$ bis zu 69,3\% in den 9 untersuchten Kategorien. Als Weiterentwicklung empfahlen die Kollegen die Bereitstellung sämtlicher medizinscher Unterlagen, da während der Untersuchung nur die aktuellen Beschwerden mittels FAX dem Tele-Konsiliar zur Verfügung gestellt werden konnte.

Eine Dysphagie nach Behandlung von Kopf-Hals-Tumoren wurde retrospektiv in einer indischen Kohorte beurteilt, bei der den lokal tätigen Ärzten Experten im Sinne einer Telekonsils mittels Videokonferenz zugeschaltet wurden. Bei Veränderung der Behandlung von 8/26 Patienten durch die telemedizinisch tätigen Kollegen zeigte sich eine signifikante Verbesserung der Dysphagie, gemessen mittels einer Skala (Functional Oral Intake Scale) von 1,46 $\pm 0,989$ auf 3,92 $\pm 1.809(p<0,0001)$. Bei Limitation wegen Fehlens einer Kontrollgruppe zeigt sich die Einholung einer Zweitmeinung als möglicherweise positiv für das Dysphagiemanagement. Ferner wurde über eine zeitlich asynchrone telemedizinische Betreuung zur intensivierten Schlucktherapie bei Patienten mit Kopf-Hals-Tumoren im heimatlichen Umfeld berichtet [157]. Dies erleichtert den Zugang zur Therapie, die durch Videokonferenz im Bedarfsfalle intensiviert werden könnte, um auf spezifische Fragestellungen einzugehen.

Insgesamt liegen damit für die verschiedenen Bereiche der Phoniatrie zahlreiche Hinweise vor, dass telemedizinische Verfahren helfen können, dieses Spezialwissen zu verbreiten und zum Wohle der Patienten besser zugänglich zu machen.

\section{Laryngologie}

Die Anfänge der Telediagnostik in der Laryngologie bestanden in der Übertragung von Bildern in Respirations- und Phonationsstellung, die versandt und überprüft wurden [158]. Dabei zeigten sich bei 29 Patienten identische Diagnosen bei Befundung durch 3 verschiedene Laryngologen. Es gab explizit keine Informationen zu einer Verblindung, wohl aber wurde auf eine voneinander unabhängige Beurteilung hingewiesen. Die Arbeit bestätigte damit die technisch ausreichende Übertragungsqualität in einem Netzwerk aus HNO-Ärzten. Bemerkenswert ist dies insoweit, als dass in dieser Kohorte auch 2 Patienten mit Larynxkarzinomen eingeschlossen wurden. Weitere Indikationen umfassten eine Rekurrensparese $(\mathrm{N}=4)$ und morphologische Veränderungen an den Stimmbändern, die aber nicht detailliert im Artikel dargestellt werden.

In einer anderen prospektiven Studie [159] wurde die flexible Endoskopie bei 79 Patienten mit einem Smartphone mittels Adapter aufgenommen. Die Durchführung erfolgte von einem Assistenzarzt und die Beurteilung durch einen Oberarzt, also fachgleich. Hierbei zeigte sich eine Übereinstimmungsrate von 88,6\% (Kappa 0,747 (95\% Cl: 0,643-0,851). In 12 Fällen wurde das klinische Vorgehen nach erneuter Bewertung geändert, eine Untersuchung musste wiederholt werden.

Insgesamt zeigen die wenigen laryngologischen Studien die Komplexität dieses Teils der HNO-ärztlichen Untersuchungstechnik auch bei Einsatz von flexiblen Nasopharyngoskopen. Daher scheint dieser Teilbereich für die Telemedizin allenfalls in fachgleichen Netzwerken und nach entsprechender Schulung zugänglich zu sein.

\section{Rhinologie}

Rhinologische Telemedizin ist im Vergleich zu anderen Bereich der HNO-Heilkunde deutlich weniger entwickelt. So wurden zwar für Patienten mit chronischer Rhinosinusitis telemedizinische Ansätze als Strategie zur Kostenreduktion und Minderung der Belastung vorgeschlagen [160]. Auch wurde mittels Smartphone eine Software präsentiert, die eine nasale Obstruktion durch Analyse der Atemgeräusche identifizieren soll und Patienten mit nasaler Obstruktion von Patienten nach einer Septumplastik unterscheiden kann [161]. Daten zur klinischen Anwendung fehlen jedoch.

Als Ansatz zur Zweitmeinung und für die Forschung würden endonasal-endoskopische Videoaufnahmen zu 2 verschiedenen Aspekten validiert:

So ist die reliable Beurteilung der endonasalen Anatomie bei chronisch-polypöser Rhinosinusitis mit Messung der Polypengröße $[162,163]$ im Rahmen von Therapiestudien ebenso möglich, wie eine reliable verblindete Bewertung von endnasaler Sekretion [162] und Ödem [162, 164]. Die Einschätzung einer Obstruktion durch eine Septumdeviation war dagegen nur eingeschränkt zuverlässig. Die Ergebnisse zur Beurteilung der mittleren Nasenmuschel waren widersprüchlich $[163,165]$, besserten sich aber bei entsprechender Schulung [165].

Ferner kann der intraoperative Aspekt der endonasalen Blutungsintensität im Rahmen von Nasennebenhöhlenoperationen standardisiert anhand von Videoaufnahmen reliabel bewertet werden [166-169]. Dank der Entwicklung der 3D-Endoskopie scheinen sich hier für eine Zweitbeurteilung interessante neue Aspekte zu ergeben [170]. Allgemein hat der Symptomkomplex der nasalen Obstruktion bislang noch keinen vielversprechenden telemedizinischen Zugang erfahren.

\section{Allergologie}

Auch für den Bereich der Allergologie wird die Telemedizin eingesetzt. So wurde die Durchführung eines Prick-Testes mit Penicillin bei Verdacht auf das Vorliegen einer Penizillinallergie beschrieben [171]. Das Ziel war, die laufende Antibiotikatherapie zu deeskalie- 
ren. Bei den 50 eingeschlossenen Patienten führte eine MTA vor Ort unter telemedizinischer Verbindung zu einem Allergologen den Test durch, der bei 46/50 Patient negativ ausfiel. Entsprechend konnten 33 Patienten erfolgreich auf ein Beta-Laktam-Antibiotikum umgestellt werden. Die 4 reagierenden Patienten erlitten keine ernsthaften Komplikationen. Aufgrund des sehr seltenen Risikos einer anaphylaktischen Reaktion bei Durchführung einer Prick-Testung $[172,173]$ erscheint die Durchführung in Deutschland kritikwürdig. Um derartigen Problemen zu begegnen, wurde bereits ein Positionspapier des Amerikanischen College of Allergy, Asthma \& Immunology [174] veröffentlicht, das die Sicherheit der telemedizinischen Behandlung betont. Für den direkten Arzt-Patienten-Kontakt wird eine vorbestehende Arzt-Patienten-Beziehung oder alternativ ein Live-Kontakt zum Aufbau derselben gefordert. Sollte dies nicht möglich sein, wird die Rolle des Allergologen als Tele-Konsiliar zu einem zuweisenden Kollegen vor Ort empfohlen. Leider nimmt auch in diesem Dokument die Forderung nach ausreichendem finanziellem Ausgleich einen breiten Raum ein, was einer kritischen Aufarbeitung der Chancen und Risiken telemedizinischer Methoden bei allergologischen Untersuchungen und Behandlungen entgegensteht.

Zum Indikationsspektrum der Telemedizin in der Allergologie weist eine retrospektive Untersuchung heterogene Indikationen bei $\mathrm{N}=112$ Patienten nach [175]. Bei einer Rate von 59\% Neuvorstellungen zeigte sich in diesem jungen Kollektiv (Alter: 26,9 \$15,3 Jahre) eine große Patientengruppe (ca. $20 \%$ ), die sich wegen einer allergischen Rhinitis vorstellte. Eine weitere Indikation war der Verdacht auf eine Nahrungsmittelallergie (30\%) bzw. eine Urtikaria (16\%). 19/112 Patienten (16,9\%) wurden zu einer anschließenden, persönlichen Vorstellung eingeladen. Letzlich ergaben sich keine signifikanten Unterschiede zwischen Erst- und Wiedervorstellungen, der Zahl der Verordnungen bei relevanten Einsparungen von Zeit- (200 Arbeitstage) und Kosten (\$58 000) durch die telemedizinische Methodik.

Zum Telemonitoring in der Allergologie liegt eine Fallserie von 2 Jungen vor [176], bei denen auf traditionellem Wege eine Allergie nicht klar den Beschwerden und den nachgewiesenen Sensibilisierungen (in RIST und RAST)zugeordnet werden konnte. Beide berichteten daher mittels Smartphone ihre Beschwerden im Zeitverlauf mittels Rhinoconjunctivitis Total Symptom Score (RTSS), so dass später diese Beschwerden der gemessenen Pollenbelastung zugeordnet werden konnten. Entsprechend wurde eine Allergie gegen Gräser- bzw. Olivenpollen gesichert. Natürlich wäre eine Datenerfassung auch konventionell mittels Beschwerdetagebuch möglich gewesen. Es ist aber gerade im Hinblick auf das Alter der Patienten (7- und 10-Jahre) anzunehmen, dass die Verwendung technischer Geräte sich Compliance-steigernd auswirkt.

Damit stellt die Allergologie ein sehr interessantes Gebiet dar für innovative technologische Lösungen. Da diese Patienten schon an das Führen von Symptomtagebüchern gewöhnt sind, mag der Einsatz von medizinischen „Apps“ aber noch geeigneter sein. Daher wird an dieser Stelle auf die entsprechende Übersichtsarbeit verwiesen.

\section{Kopf-Hals-Tumoren}

Hier sind telemedizinische Lösungen entwickelt worden auch als Unterstützung der Symptomkontrolle [177], zur Steigerung der Le- bensqualität [178] von Patienten mit Kopf-Hals-Tumoren, wie auch zur Früherkennung von Neubildungen im Mundraum [179]. Gemäß einer Online-Umfrage [180] besteht der größte Bedarf unterstützender Maßnahmen für Patienten mit einer Krebsdiagnose ( $N=212$ ) im Bereich der körperlichen Pflege (66\%), der Unterstützung einer gesunden Lebensweise (54\%), der sozialen (43\%) und psychologischen Hilfestellung (38\%) sowie im Hinblick auf Programme, die sich mit Lebensfragen beschäftigen (24\%). Auch wenn das Ergebnis der Umfrage Antworten nur von ca. einem Zehntel der Kopf-Hals-Tumor-Patienten zusammenfassen konnte, weist es auf die Faktoren hin, die in besonderem Maße mit der Suche nach Unterstützung assoziiert waren. Es handelt sich dabei u. a. um Probleme in Zusammenhang mit dem männlichem Geschlecht, jüngerem Lebensalter, einer Behandlung mit Chemotherapie oder kombinierter Radio-Chemotherapie und mit geringer Lebensqualität. All das sind Faktoren, die bekanntlich häufig und auch in Kombination bei Patienten mit Kopf-Hals-Tumoren vorliegen.

Inhaltlich auf diese Daten aufbauend, bot eine Studie eine telefonische psychosoziale Intervention an [181]. Da traditionell Kopf-HalsTumor-Patienten schlecht für psychosoziale Interventionen zu gewinnen sind [182], wurde evaluiert, ob mittels einer bis zu 8-maligen telefonischen Intervention ein positiver Effekt zu erreichen wäre. Bei Therapieabbruch von 8 der 24 eingeschlossenen Patienten zeigte sich insgesamt eine verbesserte Verarbeitung der negativen emotionalen und körperlichen Effekte der Grunderkrankung.

Zur Therapieplanung und -entscheidung wird der Einsatz zertifizierter Tumorzentren in Deutschland empfohlen. Telemedizinische Maßnahmen sind weltweit etabliert, sollte vor Ort nicht ausreichende Expertise vorhanden sein, um eine ausgewogene Beratung zu gewährleisten [183].

Zur frühzeitigen Erkennung einer Hörminderung aufgrund ototoxischer Nebenwirkung einer Cisplatin-basierten Chemotherapie wurde ein audiologisches Screening-Gerät (OtoID) entwickelt, das diese automatisiert erkennen konnte [184], und zwar mit einer Sensitivität von 80,6\% und einer Spezifität von 85,3\%. Diese Entwicklung ist damit ein einfacher Ansatz, um die Versorgungsqualität bei gleichzeitiger Minderung von Nebenwirkungen zu verbessern.

Bei Patienten mit Oropharynxkarzinom erfolgte als Ansatz zur Teletherapie eine randomisierte Untersuchung, in der eine Schlucktherapie durchgeführt wurde [185], entweder mit persönlicher Betreuung durch einen Therapeuten oder telemedizinisch unterstützt oder unabhängig allein durch den Patienten. Dabei erhielten alle 79 Patienten eine definitive Radio-Chemo-Therapie. Die Therapieadhärenz war in allen Gruppen mit 27 \% gering und am höchsten bei persönlicher Therapie. Tendenziell zeigte sich die telemedizinische Therapie der unabhängig durch den Patienten durchgeführten Therapie überlegen. Da die Durchführung einer Schlucktherapie mit Hilfe eines Therapeuten sehr ressourcenintensiv ist, erscheint der telemedizinische Ansatz dennoch wertvoll und sollte weiter entwickelt werden. In dieser Studie zeigte sich zudem ein aktiver Nikotinabusus bei Therapiebeginn als prädiktiver Faktor, der auf eine schlechte Therapieadhärenz hindeutet.

Zusammenfassend scheinen Patienten mit Kopf-Hals-Tumoren schwierige Klienten für telemedizinische Verfahren zu sein. Die Mehrzahl der Studien beschäftigt sich derzeit mit unseren Patienten, während diese bestrahlt werden. Möglicherweise ist ein früheres Angebot, auch zur Stärkung der Arzt-Patienten-Beziehung, 
hier hilfreicher, um die vom Patienten gewünschte Unterstützung zum beiderseitigen Nutzen anzubieten.

\section{Palliativmedizin}

Die Anwendung telemedizinischer Methoden wurde für die Palliativmedizin bei Patienten mit fortgeschrittener Tumorerkrankung prospektiv randomisiert evaluiert [186]. Dabei lagen bei den insgesamt 74 eingeschlossenen Patienten in 8 Fällen Tumorerkrankungen aus dem Kopf-Hals-Bereich vor. Die Studie evaluierte den Einfluss einer wöchentlichen Kontaktaufnahme auf telemedizinischem Wege über einen Zeitraum von 12 Wochen. Sie berichtet eine höhere Belastung mit Symptomen durch die telemedizinische Maßnahme und damit eine zusätzliche Beschwernis der Patienten. Diese Verschlechterung wurde von den Autoren einem „NoceboEffekt“" zugeordnet, der durch erhöhtes Bewusstsein von körperlichen Beschwerden diese noch verstärken. Allerdings behindern Protokollabweichungen in der Rekrutierung und relativ hohe Dropout-Raten die Diskussion dieser Studie. Daher sollte sie als erste randomisierte Studie ihrer Art nicht Anlass geben, einen telemedizinischen Ansatz definitiv abzulehnen [187], sondern vielmehr auf die Probleme in der Studiendurchführung in der Palliativmedizin aufmerksam machen. Zudem steht diese Studie im Widerspruch zu einer Cochrane Analyse [188], die bei Auswertung von 23 Studien, davon einer laufenden Untersuchung mit telemedizinscher Funktionalität [189], eine Effektivität für Palliativmedizin im häuslichen Umfeld bestätigen konnte. Dabei waren auch palliative Patienten mit Kopf-Hals-Tumoren in die Datenauswertung eingeschlossen.

Eine andere Untersuchung [190] konnte bei Einschluss von 18 Patienten, davon 2 mit Kopf-Hals-Tumoren, dagegen positive Effekte nachweisen. Einschlusskriterien war hier eine starke funktionelle Einschränkung (Karnofsky-Index $<60 \%$ ) und eine prognostizierte Lebenserwartung < 3 Monate. Das Angebot eines leicht herstellbaren, telemedizinischen Kontaktes mit Palliativmedizinern konnte Barrieren reduzieren, gab den Patienten das Gefühl von mehr Selbstbestimmung und erlaubte durch eine Videokonferenz auch non-verbale Kommunikation, um so nicht alle Beschwerden benennen zu müssen und so weiter bewusst werden zu lassen. Auch aus der Sicht der Betreuenden war so ein verbesserter Einblick in die momentane häusliche Situation möglich. Die Patienten und Betreuenden wertschätzten den vertieften persönlichen Kontakt und den positiven Charakter, sich zu einem neuen Gespräch verabreden zu können.

Entsprechend benennt eine Übersichtsarbeit eine ganze Reihe von Entwicklungsprojekten, um die Palliativmedizin speziell bei Kopf-Hals-Tumor-Patienten mit Hilfe telemedizinischer Verfahren weiter zu entwickeln [191].

\section{Telemedizinische Methoden in der Schlafmedizin}

Für den Bereich der Diagnostik und Therapie, speziell dem Monitoring, von Schlaf-bezogenen Atemstörungen zeigen sich eine Reihe von Entwicklungen, die aber noch keinen klaren klinischen Einsatz vorweisen können [192, 193]. So wurden im Rahmen eines Positionspapieres sowohl für die USA [194], wie für Deutschland [195], Rahmenbedingungen für telemedizinische Verfahren definiert.

\section{Telediagnostik}

Beim Vergleich einer konventionellen Polygraphie zu einer mit telemedizinischer Anbindung konnte durch das telemedizinische Verfahren keine qualitative diagnostische Verbesserung erreicht werden [196]. Es zeigte sich jedoch eine sehr hohe Reliabilität sowohl für die Anzahl von Apnoen/Hypopnoen (ICC: 0,94 (0,88-0,96)), den Apnoe-Hypopnoe-Index (ICC: 0,97 (0,95-0,98)), die Dauer von Sauerstoffentsättigungen (ICC: $0,98(0,97-0,99)$ ), als auch die durchschnittliche Sauerstoff-Sättigung (ICC: 0,97 (0,94-0,98)).

Die Kombination von Smartphone mit einem Adapter zur Messung von Sauerstoffsättigung und Puls [197] ist gut validiert. Sowohl für die Anwendung bei Kindern [198], als auch Erwachsenen [199] liegen Daten zur Anwendung als Screening mit ähnlichen Systemen vor. Dabei konnte für 15 Erwachsene eine Sensitivität der Technik von $100 \%$ und eine Spezifität von $85 \%$ festgestellt werden.

\section{Telemonitoring}

Im Bereich der schlafbezogenen Atemstörungen werden telemedizinische Methoden v. a. zum Telemonitoring eingesetzt. Ziel ist die Erhöhung der Compliance [194] v. a. der Beatmungstherapie (continous positive airway pressure [CPAP] oder biphasic positive airway pressure [BiPAP]). Die Akzeptanz auf Seite der Patienten scheint für ein Monitoring ausreichend zu sein. So zeigten sich bei Befragung von 160 Patienten 78 \% einem Monitoring offen gegenüber, aber mit $40 \%$ betrachtete auch ein relevanter Patientenanteil dieses Monitoring als zudringlich [200].

Die Effekte eines Monitorings sind bislang nicht einheitlich geklärt. Während 10 Monate nach Einleitung der Beatmungstherapie $78 \%$ der deutschen Kohorte weiterhin so überwacht wurden, ohne dass dies zu einem günstigen Therapieeffekt führte, war eine Ablehnung des Monitorings mit einem erhöhten Therapieabbruch assoziiert [200]. Während in dieser Studie das Monitoring nicht zu einer verbesserten durchschnittlichen Anwendungsdauer der CPAP-Therapie führte, konnte in weiteren Studien durch Telemonitoring die Therapieabbruchrate reduziert [201-203], der Therapiegebrauch [202-206] gesteigert und die Therapieeffizienz [207, 208], auch durch Reduktion der Arbeitszeit betreuender Mitarbeiter des Gesundheitswesen [209], verbessert werden.

\section{Teletherapie}

Aufbauend auf diesen Monitoring-Daten kann aber auch mit einem zusätzlichen günstigen Effekt therapeutisch interveniert werden: So konnte eine vergleichende, deutsche Untersuchung an insgesamt 1000 Patienten [210] bei telemedizinscher Betreuung beider Gruppen einen günstigen Effekt nachweisen, wenn zusätzlich auf einen Abfall des Beatmungsgerätgebrauches reagiert wurde. Gemäß Studienprotokoll erhielten die Teilnehmer dieser Gruppe einen Telefonanruf, wenn in den ersten 2 Wochen der Therapie die Beatmungstherapie weniger als $4 \mathrm{~h} / \mathrm{Nach}$ angewendet wurde. Diese Intervention führte zu einem erhöhten Gebrauch der Beatmungsgeräte sowohl in Stunden pro Nacht, als auch in der Anzahl der Therapietage und einer signifikant niedrigeren Rate an Therapieabbrüchen. Außerdem konnte die Häufigkeit von Schlafmaskenassoziierten Beschwerden reduziert werden.

Damit erscheint zusammenfassend im Bereich der Schlafmedizin allgemein die Entwicklung von telemedizinischen Verfahren so 
weit vorangeschritten zu sein, dass die Integration von Wünschen und Vorstellungen des jeweiligen Patienten in den diagnostischen Algorithmus einerseits und das Monitoring der Patienten zur Erhöhung der Compliance und Identifikation von Risikopatienten und zur Verbesserung des Zugangs zur Therapie mit telemedizinischen Methoden andererseits optimiert werden können.

\section{Schlussfolgerung und Ausblick}

Die Entwicklung telemedizinischer Methoden in der Hals-NasenOhrenheilkunde eröffnet einen völlig neuen Ansatz, diagnostische und therapeutische Verfahren zu hinterfragen und durch vergleichende Untersuchungen die Evidenz unseres Fachgebietes zu verbessern. Die dargestellten wissenschaftlichen Studien haben ausgereicht, um eine Re-Finanzierung der Videosprechstunde des eHealth-Gesetzes auch für die HNO-Heilkunde durchzusetzen. Dies stellt eine Chance dar, im Wettbewerb mit anderen Fachgebieten und in Kooperation mit Medizintechnik und Firmen der Informationstechnologie unser Fach im digitalen Zeitalter entsprechend modern zu gestalten, seine Attraktivität auch für jüngere Kollegen zu erhöhen und die Versorgungsqualität, speziell auch durch verbesserte Erreichbarkeit der HNO-Ärzte für jeden Patienten, zu verbessern.

\section{Interessenkonflikt}

Die Autoren geben an, dass kein Interessenkonflikt besteht.

Literatur

[1] Bundesärztekammer. Telemedizin. 2018 https://www.bundesaerztekammer.de/aerzte/telematiktelemedizin/telemedizin/

[2] Bundesärztekammer. Telemedizinische Methoden in der Patientenversorgung - Begriffliche Verortung. 2015, DOI: https://www. bundesaerztekammer.de/fileadmin/user_upload/downloads/ pdf-Ordner/Telemedizin_Telematik/Telemedizin/Telemedizinische_ Methoden_in_der_Patientenversorgung_Begriffliche_Verortung.pdf

[3] Field M]. Telemedicine: A Guide to Assessing Telecommunications in Health Care. Washington (DC): 1996

[4] Amenta F. The Centro Internazionale Radio Medico Symposium "The Way Forward of Maritime Telemedicine". Int Marit Health 2016; 67: 56

[5] Mahdi SS, Amenta F. 80 years of CIRM. A journey of commitment and dedication in providing maritime medical assistance. Int Marit Health 2016; 67: 187-195

[6] Westlund K, Attvall S, Nilsson R et al. Telemedical Maritime Assistance Service (TMAS) to Swedish merchant and passengers ships 1997-2012. Int Marit Health 2016; 67: 24-30

[7] Burg G.. Telemedicine and Teledermatology. Curr Probl Dermatol 2003; 32:

[8] Hickel W], Mala TA. A history of health and social services in Alaska. Frontier Printing in Anchorage. Alaska: Department of Health and Social Services; 1993

[9] Hendel S, Duncan T. Broad Horizons: A History of Australian Aeromedical Retrieval. Int Anesthesiol Clin 2018; 56: 34-46

[10] Langford SA. The Royal Flying Doctor Service of Australia. Its foundation and early development. Med J Aust 1994; 161: 91-94

[11] Hays H, Carroll M, Ferguson S et al. The Success of Telehealth Care in the Indian Health Service. Virtual Mentor 2014; 16: 986-996
[12] Hofstetter PJ, Kokesh J, Ferguson AS et al. The impact of telehealth on wait time for ENT specialty care. Telemed J E Health 2010; 16: 551-556

[13] Kokesh J, Ferguson AS, Patricoski C et al. Digital images for postsurgical follow-up of tympanostomy tubes in remote Alaska. Otolaryngol Head Neck Surg 2008; 139: 87-93

[14] Patricoski C, Kokesh J, Ferguson AS et al. A comparison of in-person examination and video otoscope imaging for tympanostomy tube follow-up. Telemed J E Health 2003; 9: 331-344

[15] Kokesh J, Ferguson AS, Patricoski C. The Alaska experience using store-and-forward telemedicine for ENT care in Alaska. Otolaryngol Clin North Am 2011; 44: 1359-1374 ix

[16] World Medical Association. WMA Statement on Access to Health Care; Adopted by the 40th World Medical Assembly, Vienna, Austria, September 1988 and revised by the 57th WMA General Assembly, Pilanesberg, South Africa, October 2006 and by the 68th WMA General Assembly, Chicago, United States, October 2017. 2006 https://www.wma.net/policies-post/wma-statement-on-access-tohealth-care/

[17] Association WM. WMA Statement on the Ethics of Telemedicine Adopted by the 58th WMA General Assembly, Copenhagen, Denmark, October 2007. 2007 https://www.wma.net/policies-post/ wma-statement-on-the-ethics-of-telemedicine/

[18] Bundesärztekammer. 1. Voraussetzungen für gute Telemedizin. 2010 https://www.bundesaerztekammer.de/aerztetag/beschlussprotokolleab-1996/113-daet-2010/top-v/telemedizin/1-voraussetzungen/

[19] Perez-Rodriguez G, Brito-Zurita OR, Sistos-Navarro E et al. El seguimiento telemetrico disminuye la visita a la sala de urgencias y coste de atencion en enfermos con insuficiencia cardiaca cronica. Cir Cir 2015; 83: 279-285

[20] Scherr D, Kastner P, Kollmann A et al. Effect of home-based telemonitoring using mobile phone technology on the outcome of heart failure patients after an episode of acute decompensation: randomized controlled trial. J Med Internet Res 2009; 11: e34

[21] Zhai YK, Zhu W], Hou HL et al. Efficacy of telemedicine for thrombolytic therapy in acute ischemic stroke: a meta-analysis. J Telemed Telecare 2015; 21: 123-130

[22] Demaerschalk BM, Raman R, Ernstrom K et al. Efficacy of telemedicine for stroke: pooled analysis of the Stroke Team Remote Evaluation Using a Digital Observation Camera (STRokE DOC) and STRokE DOC Arizona telestroke trials. Telemed J E Health 2012; 18: 230-237

[23] Audebert HJ, Schultes K, Tietz V et al. Long-term effects of specialized stroke care with telemedicine support in community hospitals on behalf of the Telemedical Project for Integrative Stroke Care (TEMPiS). Stroke 2009; 40: 902-908

[24] Meyer BC, Raman R, Hemmen T et al. Efficacy of site-independent telemedicine in the STRokE DOC trial: a randomised, blinded, prospective study. Lancet Neurol 2008; 7: 787-795

[25] Waller M, Stotler C. Telemedicine: a Primer. Curr Allergy Asthma Rep 2018; 18: 54

[26] Bashshur R, Shannon G, Krupinski E et al. The taxonomy of telemedicine. Telemed J E Health 2011; 17: 484-494

[27] Fischer F, Aust V, Krämer A. eHealth: Hintergrund und Begriffsbestimmung. In: Fischer F, Krämer A, (Hrsg.). eHealth in Deutschland. Springer-Verlag Berlin Heidelberg; 2016

[28] Ekeland AG, Bowes A, Flottorp S. Effectiveness of telemedicine: a systematic review of reviews. Int J Med Inform 2010; 79: 736-771

[29] Center for Connected Health Policy. 50 State Scan of Telehealth Reimbursement Laws and Medicaid Policies - Factsheet. 2018 http:// www.cchpca.org/sites/default/files/resources/50\%20State \%20 Factsheet \%20Spring \%202018\%20FINAL.pdf 
[30] Wicklund E.CMS proposes more medicare reimbursement for telehealth, RPM. 2018 https://mhealthintelligence.com/news/ cms-proposes-more-medicare-reimbursement-for-telehealth-rpm

[31] Pune, Maharashtra. Telehealth Market worth 9.35 Billion USD by 2021. 2018 https://www.marketsandmarkets.com/PressReleases/telehealth.asp

[32] Ungeklärte Finanzierung bremst Telemedizin. Deutsches Ärzteblatt 2011; 2: 14

[33] GKV-Spitzenverband. Telemedizin in der vertragsärztlichen Versorgung. 2016 https://www.gkv-spitzenverband.de/media/dokumente/ presse/publikationen/Positionspapier_Telemedizin_03-2016.pdf

[34] Bundestag D. Gesetz für sichere digitale Kommunikation und Anwendungen im Gesundheitswesen sowie zur Änderung weiterer Gesetze. Bundesgesetzblatt 2015; I: 2408-2423

[35] GKV-Spitzenverband, Bundesvereinigung K. Vereinbarung über die Anforderungen an die technischen Verfahren zur Videosprechstunde gemäß §291g Absatz 4 SGB V. 2016 http://www.kbv.de/media/sp/ Anlage_31b_Videosprechstunde.pdf

[36] Bundesvereinbarung K. Anbieter für Videosprechstunde zertifiziert. 2017 http://www.kbv.de/html/1150_30410.php

[37] Ärzteblatt.de. Vergütung für Videosprechstunde festgelegt. 2018 https://www.aerzteblatt.de/nachrichten/73320/Verguetung-fuerVideosprechstunde-festgelegt

[38] McCool RR, Davies L. Where Does Telemedicine Fit into Otolaryngology? An Assessment of Telemedicine Eligibility among Otolaryngology Diagnoses. Otolaryngol Head Neck Surg 2018; 158: 641-644

[39] Okoroafor IJ, Chukwuneke FN, Ifebunandu $\mathrm{N}$ et al. Telemedicine and biomedical care in Africa: Prospects and challenges. Niger J Clin Pract 2017; 20: 1-5

[40] Russo JE, McCool RR, Davies L. VA Telemedicine: An Analysis of Cost and Time Savings. Telemed J E Health 2016; 22: 209-215

[41] Locatis C, Ackerman M. Three principles for determining the relevancy of store-and-forward and live interactive telemedicine: reinterpreting two telemedicine research reviews and other research. Telemed J E Health 2013; 19: 19-23

[42] Pötzsch I, Rößger F. Bevölkerung Deutschlands bis 2060; 13. koordinierte Bevölkerungsvorausberechnung: Statistisches Bundesamt. 2015

[43] Bush ML, Hardin B, Rayle C et al. Rural barriers to early diagnosis and treatment of infant hearing loss in Appalachia. Otol Neurotol 2015; 36: 93-98

[44] Holderried M, Holderried F, Tropitzsch A. eHealth in otorhinolaryngology: a disruptive innovation. Eur Arch Otorhinolaryngol 2017; 274: 4071-4072

[45] Kmucha ST. Physician liability issues and telemedicine: Part 3 of 3. Ear Nose Throat J 2016; 95: 12-14

[46] Kmucha ST. Physician liability issues and telemedicine: Part 1 of 3. Ear Nose Throat J 2015; 94: 428-429

[47] Kmucha ST. Physician liability issues and telemedicine: Part 2 of 3. Ear Nose Throat J 2015; 94: 466-469

[48] Christian D, Feussner H, Wienke A. Rechtsfragen der Telemedizin. Berlin Heidelberg: Springer-Verlag; 2001

[49] Bundesärztekammer. Hinweise und Erläuterungen zu § 7 Absatz 4 MBO-Ä (Fernbehandlung). 2015 https://www.bundesaerztekammer. de/fileadmin/user_upload/downloads/pdf-Ordner/Recht/2015-1211_Hinweise_und_Erlaeuterungen_zur_Fernbehandlung.pdf

[50] Bundesärztekammer. Synopse zur Änderung § 7 Abs. 4 MBO-Ä (Fernbehandlung). 2018, DOI:

[51] Baden-Württemberg Lv. Drucksache 16 / 3161 Antrag der Abg. Rainer Hinderer u. a. SPD und Stellungnahme des Ministeriums für Soziales und Integration „Den rechtlichen Rahmen für die Fernbehandlung/Telemedizin den Behandlungsmöglichkeiten der Gegenwart anpassen“. 2017 https://www.landtag-bw.de/files/live/sites/LTBW/ files/dokumente/WP16/Drucksachen/3000/16_3161_D.pdf
[52] Baden-Württemberg Lv. Drucksache 16 /3731 Beschlussempfehlungen und Berichte der Fachausschüsse zu Anträgen von Fraktionen und von Abgeordneten. 2017 https://www.landtag-bw.de/files/live/sites/ LTBW/files/dokumente/WP16/Drucksachen/3000/16_3731_D.pdf

[53] Kassenärztliche Bundesvereinigung, GKV-Spitzenverband (Spitzenverband Bund der Krankenkassen). Bundesmantelvertrag - Ärzte vom 1. Juli 2018 https://www.kbv.de/media/sp/BMV_Aerzte.pdf

[54] Bundesvereinigung K, Krankenkassen (G-SSBd. Bundesmantelvertrag - Ärzte vom 1. Juli 2018 2018. https://www.kbv.de/media/sp/ BMV_Aerzte.pdf

[55] Ärztetag D. Beschlussprotokoll. 2018 https://www.bpm-ev.de/ images/121._D\%C3\%84T_Beschlussprotokoll.pdf

[56] Bund H. Das Fernbehandlungsverbot - die wichtigsten Fakten im Überblick. Hartmannbund Magazin 2018; 2: 18-19

[57] Sailer R, Winke A. Telemedizin: Ja, aber!. GMS Mitteilungen aus der AWMF 2016; 1: Doc2

[58] Bergmann KO. Telemedizin und E-Health-Gesetz - neue Haftungsgefahren? N E T L E T T E R 2016; 1

[59] Yulzari R, Bretler S, Avraham Y et al. Mobile Technology-Based Real-Time Teleotolaryngology Care Facilitated by a Nonotolaryngologist Physician in an Adult Population. Ann Otol Rhinol Laryngol 2018; 127: 46-50

[60] Campisano F, Ozel S, Ramakrishnan A et al. Towards a soft robotic skin for autonomous tissue palpation. IEEE International Conference on Robotics and Automation 2017 doi:10.1109/ICRA.2017.7989729 6150-6155

[61] Pacchierotti C, Sinclair S, Solazzi M et al. Wearable Haptic Systems for the Fingertip and the Hand: Taxonomy, Review, and Perspectives. IEEE Trans Haptics 2017; 10: 580-600

[62] van den Berg N, Schmidt S, Stentzel U et al. Telemedizinische Versorgungskonzepte in der regionalen Versorgung ländlicher Gebiete : Möglichkeiten, Einschränkungen, Perspektiven. Bundesgesundheitsblatt Gesundheitsforschung Gesundheitsschutz 2015; 58: 367-373

[63] Holderried M, Ernst C, Holderried F et al. The potential of eHealth in otorhinolaryngology-head and neck surgery: patients' perspectives. Eur Arch Otorhinolaryngol 2017; 274: 2933-2943

[64] Saffarzadeh AG, Verma SP, Billimek J. Internet Usage and eHealth Literacy of Patients in an Otolaryngology - Head and Neck Surgery Clinic. Otolaryngology-Head and Neck Surgery 2014; 151: 149

[65] Mergenthal K, Leifermann M, Beyer M et al. Delegation hausarztlicher Tatigkeiten an qualifiziertes medizinisches Fachpersonal in Deutschland - eine Ubersicht. Gesundheitswesen 2016; 78: e62-e68

[66] Mergenthal K, Guthlin C, Beyer M et al. Wie bewerten und akzeptieren Patienten die Betreuung durch Medizinische Fachangestellte in der Hausarztpraxis? Ergebnisse einer Patienten-Befragung in der $\mathrm{HzV}$ in Baden-Württemberg. Gesundheitswesen 2016, doi:10.1055/s-0042-110402

[67] Mergenthal K, Beyer M, Gerlach FM et al. Sharing Responsibilities within the General Practice Team - A Cross-Sectional Study of Task Delegation in Germany. PLoS One 2016; 11: e0157248

[68] Laux G, Szecsenyi J, Mergenthal K et al. Hausarztzentrierte Versorgung in Baden-Württemberg: Ergebnisse einer qualitativen und querschnittlich quantitativen Evaluation. Bundesgesundheitsblatt Gesundheitsforschung Gesundheitsschutz 2015; 58: 398-407

[69] Mergenthal K, Beyer M, Guthlin C et al. Evaluation des VERAHEinsatzes in der Hausarztzentrierten Versorgung in BadenWürttemberg. Z Evid Fortbild Qual Gesundhwes 2013; 107: 386-393

[70] Gerlach FM, Szecsenyi ]. Hausarztzentrierte Versorgung in Baden-Württemberg - Konzept und Ergebnisse der kontrollierten Begleitevaluation. Z Evid Fortbild Qual Gesundhwes 2013; 107: 365-371 
[71] Fiss T, Schaefer M, van den Berg N et al. Zeitbedarf und Kosten fur die Umsetzung eines Medikamentenreviews und assoziierter pharmazeutischer und medizinischer Evaluation im ambulanten Versorgungssektor. Gesundheitswesen 2012; 74: 322-327

[72] van den Berg N, Meinke C, Hoffmann W. Möglichkeiten und Grenzen der Telemedizin in der Flächenversorgung. Ophthalmologe 2009; 106: 788-794

[73] van den Berg N, Fiss T, Meinke C et al. GP-support by means of AGnES-practice assistants and the use of telecare devices in a sparsely populated region in Northern Germany-proof of concept. BMC Fam Pract 2009; 10: 44

[74] Beule AG. Telemedizinische HNO-Arzt-Konsultation. HNO 2013; 61: 494

[75] Gollnick I, Frehiwot M, Krause EM et al. Interdisziplinäre Telekonsultation: Erste praktische Erfahrungen mit 100 Patienten. HNO 2013; 61: 495-503

[76] Holderried M, Hoeper A, Holderried F et al. Attitudes Toward e-Health: The Otolaryngologists' Point of View. Telemed J E Health 2018; 24: 425-432

[77] Williams AM, Bhatti UF, Alam HB et al. The role of telemedicine in postoperative care. Mhealth 2018; 4: 11

[78] Rimmer RA, Christopher V, Falck A et al. Telemedicine in otolaryngology outpatient setting-single Center Head and Neck Surgery experience. Laryngoscope 2018, doi:10.1002/lary.27123

[79] Totty JP, Harwood AE, Wallace T et al. Use of photograph-based telemedicine in postoperative wound assessment to diagnose or exclude surgical site infection. J Wound Care 2018; 27: 128-135

[80] Wilson AP, Treasure T, Sturridge MF et al. A scoring method (ASEPSIS) for postoperative wound infections for use in clinical trials of antibiotic prophylaxis. Lancet 1986; 1: 311-313

[81] Sclafani AP, Heneghan C, Ginsburg J et al. Teleconsultation in otolaryngology: live vs. store and forward consultations. Otolaryngol Head Neck Surg 1999; 120: 62-72

[82] Jodkowska A, Hosemann W, Beule A. Reliabilität HNO-ärztlicher Beurteilungen als Telemedizinisches Konsil. 2011;https://www.egms. de/static/de/meetings/hnod2011/11hnod124.shtml

[83] Akhtar M, Van Heukelom PG, Ahmed A et al. Telemedicine Physical Examination Utilizing a Consumer Device Demonstrates Poor Concordance with In-Person Physical Examination in Emergency Department Patients with Sore Throat: A Prospective Blinded Study. Telemed J E Health 2018, doi:10.1089/tmj.2017.0240

[84] Ullah R, Gilliland D, Adams D. Otolaryngology consultations by real-time telemedicine. Ulster Med J 2002; 71: 26-29

[85] Moberly AC, Zhang M, Yu L et al. Digital otoscopy vs. microscopy: How correct and confident are ear experts in their diagnoses? J Telemed Telecare 2018; 24: 453-459

[86] Kokesh J, Ferguson AS, Patricoski C. Telehealth in Alaska: delivery of health care services from a specialist's perspective. Int J Circumpolar Health 2004; 63: 387-400

[87] Kokesh J, Ferguson AS, Patricoski C. Preoperative planning for ear surgery using store-and-forward telemedicine. Otolaryngol Head Neck Surg 2010; 143: 253-257

[88] Mbao MN, Eikelboom RH, Atlas MD et al. Evaluation of videootoscopes suitable for tele-otology. Telemed J E Health 2003; 9: 325-330

[89] Seim NB, Philips RHW, Matrka LA et al. Developing a synchronous otolaryngology telemedicine Clinic: Prospective study to assess fidelity and diagnostic concordance. Laryngoscope 2018; 128 : 1068-1074

[90] Biagio L, Swanepoel de W, Laurent C et al. Video-otoscopy recordings for diagnosis of childhood ear disease using telehealth at primary health care level. J Telemed Telecare 2014; 20: 300-306
[91] Gupta N, Chawla N, Gupta D et al. Community triage of otology patients using a store-and-forward telemedicine device: A feasibility study. Ear Nose Throat J 2017; 96: 246-249

[92] McCarthy M, Munoz K, White KR. Teleintervention for infants and young children who are deaf or hard-of-hearing. Pediatrics 2010; 126: (Suppl 1): S52-S58

[93] Pereira O, Pasko LE, Supinski J et al. Is there a clinical application for tablet-based automated audiometry in children? Int J Pediatr Otorhinolaryngol 2018; 110: 87-92

[94] Levy DA, Hill DR, Bia FJ et al. Tablet-based Hearing Screening in Children Aged 5 to 17 in Rural Dominican Republic. Otol Neurotol 2018; 39: 823-828

[95] Kelly EA, Stadler ME, Nelson S et al. Tablet-based Screening for Hearing Loss: Feasibility of Testing in Nonspecialty Locations. Otol Neurotol 2018; 39: 410-416

[96] Dillon H, Mee C, Moreno JC et al. Hearing tests are just child's play: the sound scouts game for children entering school. Int J Audiol 2018; 57: 529-537

[97] Samelli AG, Rabelo CM, Sanches SGG et al. Tablet-Based Hearing Screening Test. Telemed J E Health 2017; 23: 747-752

[98] Harris MS, Dodson EE. Hearing health access in developing countries. Curr Opin Otolaryngol Head Neck Surg 2017; 25: 353-358

[99] Skarzynski PH, Swierniak W, Pilka A et al. A Hearing Screening Program for Children in Primary Schools in Tajikistan: A Telemedicine Model. Med Sci Monit 2016; 22: 2424-2430

[100] Elpers ], Lester C, Shinn JB et al. Rural Family Perspectives and Experiences with Early Infant Hearing Detection and Intervention: A Qualitative Study. J Community Health 2016; 41: 226-233

[101] Molander P, Nordqvist P, Oberg M et al. Internet-based hearing screening using speech-in-noise: validation and comparisons of self-reported hearing problems, quality of life and phonological representation. BMJ Open 2013; 3: e003223

[102] Delb W, Merkel D, Pilorget K et al. Effectiveness of a TEOAE-based screening program. Can a patient-tracking system effectively be organized using modern information technology and central data management? Eur Arch Otorhinolaryngol 2004; 261: 191-196.

[103] Givens GD, Elangovan S. Internet application to tele-audiology-"nothin' but net". Am J Audiol 2003; 12: 59-65

[104] Givens GD, Blanarovich A, Murphy T et al. Internet-based tele-audiometry system for the assessment of hearing: a pilot study. Telemed ] E Health 2003; 9: 375-378

[105] Swanepoel de W, Koekemoer D, Clark J. Intercontinental hearing assessment - a study in tele-audiology. J Telemed Telecare 2010; 16: 248-252

[106] Yao J], Yao D, Givens G. A Browser-Server-Based Tele-audiology System That Supports Multiple Hearing Test Modalities. Telemed J E Health 2015; 21: 697-704

[107] Smits C, Merkus P, Houtgast T. How we do it: The Dutch functional hearing-screening tests by telephone and internet. Clin Otolaryngol 2006; 31: 436-440

[108] Bexelius C, Honeth L, Ekman A et al. Evaluation of an internet-based hearing test - comparison with established methods for detection of hearing loss. J Med Internet Res 2008; 10: e32

[109] Honeth L, Bexelius C, Eriksson M et al. An internet-based hearing test for simple audiometry in nonclinical settings: preliminary validation and proof of principle. Otol Neurotol 2010; 31: 708-714

[110] Blaiser KM, Behl D, Callow-Heusser C et al. Measuring Costs and Outcomes of Tele-Intervention When Serving Families of Children who are Deaf/Hard-of-Hearing. Int J Telerehabil 2013; 5: 3-10

[111] Xu CQ, Smith AC, Scuffham PA et al. A cost minimisation analysis of a telepaediatric otolaryngology service. BMC Health Serv Res 2008; 8: 30 
[112] Lancaster P, Krumm M, Ribera J et al. Remote hearing screenings via telehealth in a rural elementary school. Am J Audiol 2008; 17: 114-122

[113] Smith AC, Armfield NR, Wu WI et al. A mobile telemedicine-enabled ear screening service for Indigenous children in Queensland: activity and outcomes in the first three years. J Telemed Telecare 2012; 18: 485-489

[114] Smith AC, Brown C, Bradford N et al. Monitoring ear health through a telemedicine-supported health screening service in Queensland. J Telemed Telecare 2015; 21: 427-430

[115] Smith AC, Dowthwaite S, Agnew J et al. Concordance between real-time telemedicine assessments and face-to-face consultations in paediatric otolaryngology. Med J Aust 2008; 188: 457-460

[116] Smith AC, Armfield NR, Wu WI et al. Changes in paediatric hospital ENT service utilisation following the implementation of a mobile, indigenous health screening service. J Telemed Telecare 2013; 19: 397-400

[117] Yanov Y, Kuzovkov V, Sugarova S et al. Successful application and timing of a remote network for intraoperative objective measurements during cochlear implantation surgery. Int J Audiol 2018; doi:10 .1080/14992027.2018.1479783: 1-7

[118] Shapiro WH, Huang T, Shaw T et al. Remote intraoperative monitoring during cochlear implant surgery is feasible and efficient. Otol Neurotol 2008; 29: 495-498

[119] Krüger-Brand HE. Mobile Medizintechnik: Telemedizin für die Nachsorge. Dtsch Arztebl 2013; 110: A-2014 / B-1780 / C-1742

[120] „Remote-Care“ gewinnt den Niedersächsischen Gesundheitspreis 2016; 2016

[121] Präsidium der Deutschen Gesellschaft für Hals-Nasen-Ohrenheilkunde. Weißbuch Cochlea-Implantat (CI) -Versorgung 2018

[122] Deutsche Gesllschaft für Hals-Nasen-Ohrenheilkunde K-uH. S2k-Leitlinie "Cochlea-Implantat Versorgung einschließlich zentral-auditorischer Implantate“. 2012 https://www.awmf.org/ uploads/tx_szleitlinien/017-071I_S2k_Cochlea_Implant_ Versorgung_2012-05-abgelaufen.pdf

[123] DGHNO Pd. Weißbuch Cochlea-Implantat(Cl)-Versorgung 2018

[124] Guitton MJ. Telemedicine in tinnitus: feasibility, advantages, limitations, and perspectives. ISRN Otolaryngol 2013; 2013: 218265

[125] Andersson G, Kaldo V. Internet-based cognitive behavioral therapy for tinnitus. J Clin Psychol 2004; 60: 171-178

[126] Andersson G, Kaldo-Sandstrom V, Strom L et al. Internet administration of the Hospital Anxiety and Depression Scale in a sample of tinnitus patients. J Psychosom Res 2003; 55: 259-262

[127] Henry JA, Zaugg TL, Myers PJ et al. Pilot study to develop telehealth tinnitus management for persons with and without traumatic brain injury. J Rehabil Res Dev 2012; 49: 1025-1042

[128] Nondahl DM, Cruickshanks KJ, Dalton DS et al. The impact of tinnitus on quality of life in older adults. J Am Acad Audiol 2007; 18: 257-266

[129] Weise C, Kleinstauber M, Andersson G. Internet-Delivered CognitiveBehavior Therapy for Tinnitus: A Randomized Controlled Trial. Psychosom Med 2016; 78: 501-510

[130] Hesser H, Gustafsson T, Lunden C et al. A randomized controlled trial of Internet-delivered cognitive behavior therapy and acceptance and commitment therapy in the treatment of tinnitus. J Consult Clin Psychol 2012; 80: 649-661

[131] Kaldo-Sandstrom V, Larsen HC, Andersson G. Internet-based cognitive-behavioral self-help treatment of tinnitus: clinical effectiveness and predictors of outcome. Am J Audiol 2004; 13: 185-192

[132] Kaldo V, Levin S, Widarsson J et al. Internet vs. group cognitive-behavioral treatment of distress associated with tinnitus: a randomized controlled trial. Behav Ther 2008; 39: 348-359
[133] Beukes EW, Allen PM, Manchaiah V et al. Internet-Based Intervention for Tinnitus: Outcome of a Single-Group Open Trial. J Am Acad Audiol 2017; 28: 340-351

[134] Beukes EW, Baguley DM, Allen PM et al. Guided Internet-based vs. face-to-face clinical care in the management of tinnitus: study protocol for a multi-centre randomised controlled trial. Trials 2017; 18: 186

[135] Beukes EW, Baguley DM, Allen PM et al. Audiologist-Guided Internet-Based Cognitive Behavior Therapy for Adults With Tinnitus in the United Kingdom: A Randomized Controlled Trial. Ear Hear 2018; 39: 423-433

[136] Beukes EW, Manchaiah V, Baguley DM et al. Process evaluation of Internet-based cognitive behavioural therapy for adults with tinnitus in the context of a randomised control trial. Int J Audiol 2018; 57: 98-109

[137] Kleinstauber M, Weise C, Andersson G et al. Personality traits predict and moderate the outcome of Internet-based cognitive behavioural therapy for chronic tinnitus. Int J Audiol 2018; 57: 538-544

[138] Probst T, Weise C, Andersson G et al. Differences in baseline and process variables between non-responders and responders in Internet-based cognitive behavior therapy for chronic tinnitus. Cogn Behav Ther 2018; doi:10.1080/16506073.2018.1476582 1-13

[139] Viirre E. Vestibular telemedicine and rehabilitation. Applications for virtual reality. Stud Health Technol Inform 1996; 29: 299-305

[140] Brandt T, Zwergal A, Jahn K et al. Institutional profile: integrated center for research and treatment of vertigo, balance and ocular motor disorders. Restor Neurol Neurosci 2010; 28: 135-143

[141] Gutierrez RO, Galan Del Rio F, Cano de la Cuerda R et al. A telerehabilitation program by virtual reality-video games improves balance and postural control in multiple sclerosis patients. NeuroRehabilitation 2013; 33: 545-554

[142] Ortiz-Gutierrez R, Cano-de-la-Cuerda R, Galan-del-Rio F et al. A telerehabilitation program improves postural control in multiple sclerosis patients: a Spanish preliminary study. Int J Environ Res Public Health 2013; 10: 5697-5710

[143] Jessiman S. Speech and language services using telehealth technology in remote and underserviced areas. J Speech Lang Pathol Audiol 2003; 27: 45-51

[144] Towey MP. Speech telepractice: installing a speech therapy upgrade for the 21st century. Int J Telerehabil 2012; 4: 73-78

[145] Sicotte C, Lehoux P, Fortier-Blanc J et al. Feasibility and outcome evaluation of a telemedicine application in speech-language pathology. J Telemed Telecare 2003; 9: 253-258

[146] Allen CR. The use of email as a component of adult stammering therapy: a preliminary report. J Telemed Telecare 2011; 17: 163-167

[147] Carey B, O'Brian S, Onslow M et al. Randomized controlled non-inferiority trial of a telehealth treatment for chronic stuttering: the Camperdown Program. Int J Lang Commun Disord 2010; 45: 108-120

[148] Prins D, Ingham RJ. Reviewing the literature: comments on "The Camperdown Program: outcomes of a new prolonged-speech treatment model" (2003) for stuttering. J Speech Lang Hear Res 2005; 48: 1025-1028 author reply 1029-1032

[149] O'Brian S, Onslow M, Cream A et al. The Camperdown Program: outcomes of a new prolonged-speech treatment model. J Speech Lang Hear Res 2003; 46: 933-946

[150] O'Brian S, Packman A, Onslow M. Telehealth delivery of the Camperdown Program for adults who stutter: a phase I trial. J Speech Lang Hear Res 2008; 51: 184-195

[151] Carey B, O'Brian S, Onslow M et al. Webcam delivery of the Camperdown Program for adolescents who stutter: a phase I trial. Lang Speech Hear Serv Sch 2012; 43: 370-380 
[152] Hearne A, Packman A, Onslow M et al. Developing treatment for adolescents who stutter: a phase I trial of the Camperdown Program. Lang Speech Hear Serv Sch 2008; 39: 487-497

[153] Carey B, O'Brian S, Lowe R et al. Webcam delivery of the Camperdown Program for adolescents who stutter: a phase II trial. Lang Speech Hear Serv Sch 2014; 45: 314-324

[154] Ingham RJ. Comments on recent developments in stuttering treatment maintenance research using the Camperdown Program. J Speech Lang Hear Res 2012; 55: 306-309 author reply 310-302

[155] Burns CL, Ward EC, Hill A] et al. A pilot trial of a speech pathology telehealth service for head and neck cancer patients. J Telemed Telecare 2012; 18: 443-446

[156] Malandraki GA, McCullough G, He X et al. Teledynamic evaluation of oropharyngeal swallowing. J Speech Lang Hear Res 2011; 54: 1497-1505

[157] Ward EC, Wall LR, Burns CL et al. Application of telepractice for head and neck cancer management: a review of speech language pathology service models. Curr Opin Otolaryngol Head Neck Surg 2017; 25: 169-174

[158] Furukawa M, Furukawa MK, Mizojiri G et al. Telemedicine in laryngology. Telemed J 1998; 4: 329-333

[159] Lozada KN, Morton K, Stepan K et al. The clinical impact of bedside fiberoptic laryngoscopic recording on a tertiary consult service. Laryngoscope 2018; 128: 818-822

[160] Yip J, Vescan AD, Witterick IJ et al. The personal financial burden of chronic rhinosinusitis: A Canadian perspective. Am J Rhinol Allergy 2017; 31: 216-221

[161] Choi H, Park IH, Yoon HG et al. Wireless patient monitoring system for patients with nasal obstruction. Telemed J E Health 2011; 17: 46-49

[162] Raithatha R, Anand VK, Mace JC et al. Interrater agreement of nasal endoscopy for chronic rhinosinusitis. Int Forum Allergy Rhinol 2012; 2: $144-150$

[163] McCoul ED, Smith TL, Mace JC et al. Interrater agreement of nasal endoscopy in patients with a prior history of endoscopic sinus surgery. Int Forum Allergy Rhinol 2012; 2: 453-459

[164] Psaltis AJ, Li G, Vaezeafshar R et al. Modification of the Lund-Kennedy endoscopic scoring system improves its reliability and correlation with patient-reported outcome measures. Laryngoscope 2014; 124: 2216-2223

[165] Colley P, Mace JC, Schaberg MR et al. Impact of educational intervention on the interrater agreement of nasal endoscopy interpretation. Laryngoscope 2015; 125: 2259-2265

[166] Jahanshahi ], Hashemian F, Pazira S et al. Effect of topical tranexamic acid on bleeding and quality of surgical field during functional endoscopic sinus surgery in patients with chronic rhinosinusitis: a triple blind randomized clinical trial. PLoS One 2014; 9: e104477

[167] Valentine R, Athanasiadis T, Moratti S et al. The efficacy of a novel chitosan gel on hemostasis and wound healing after endoscopic sinus surgery. Am J Rhinol Allergy 2010; 24: 70-75

[168] Valentine R, Athanasiadis T, Moratti $S$ et al. The efficacy of a novel chitosan gel on hemostasis after endoscopic sinus surgery in a sheep model of chronic rhinosinusitis. Am J Rhinol Allergy 2009; 23: 71-75

[169] Athanasiadis T, Beule A, Embate J et al. Standardized video-endoscopy and surgical field grading scale for endoscopic sinus surgery: a multi-centre study. Laryngoscope 2008; 118: 314-319

[170] Klapan I, Vranjes Z, Prgomet D et al. Application of advanced virtual reality and 3D computer assisted technologies in tele-3D-computer assisted surgery in rhinology. Coll Antropol 2008; 32: 217-219

[171] Staicu ML, Holly AM, Conn KM et al. The Use of Telemedicine for Penicillin Allergy Skin Testing. J Allergy Clin Immunol Pract 2018, doi:10.1016/j.jaip.2018.04.038
[172] Ring J, Beyer K, Biedermann T et al. Guideline for acute therapy and management of anaphylaxis: S2 Guideline of the German Society for Allergology and Clinical Immunology (DGAKI), the Association of German Allergologists (AeDA), the Society of Pediatric Allergy and Environmental Medicine (GPA), the German Academy of Allergology and Environmental Medicine (DAAU), the German Professional Association of Pediatricians (BVKJ), the Austrian Society for Allergology and Immunology (OGAI), the Swiss Society for Allergy and Immunology (SGAI), the German Society of Anaesthesiology and Intensive Care Medicine (DGAI), the German Society of Pharmacology (DGP), the German Society for Psychosomatic Medicine (DGPM), the German Working Group of Anaphylaxis Training and Education (AGATE) and the patient organization German Allergy and Asthma Association (DAAB). Allergo J Int 2014; 23: 96-112

[173] Rueff F, Bergmann KC, Brockow K et al. Hauttests zur Diagnostik von allergischen Soforttyp-Reaktionen. Leitlinie der Deutschen Gesellschaft fur Allergologie und klinischen Immunologie (DGAKI). Pneumologie 2011; 65: 484-495

[174] Elliott T, Shih J, Dinakar C et al. American College of Allergy, Asthma \& Immunology Position Paper on the Use of Telemedicine for Allergists. Ann Allergy Asthma Immunol 2017; 119: 512-517

[175] Waibel KH. Synchronous telehealth for outpatient allergy consultations: A 2-year regional experience. Ann Allergy Asthma Immunol 2016; 116: 571-575 e571

[176] Bianchi A, Tsilochristou O, Gabrielli F et al. The Smartphone: A Novel Diagnostic Tool in Pollen Allergy? J Investig Allergol Clin Immunol 2016; 26: 204-207

[177] Head BA, Studts JL, Bumpous JM et al. Development of a telehealth intervention for head and neck cancer patients. Telemed J E Health 2009; 15: 44-52

[178] van den Brink JL, Moorman PW, de Boer MF et al. Impact on quality of life of a telemedicine system supporting head and neck cancer patients: a controlled trial during the postoperative period at home. J Am Med Inform Assoc 2007; 14: 198-205

[179] Skandarajah A, Sunny SP, Gurpur P et al. Mobile microscopy as a screening tool for oral cancer in India: A pilot study. PLoS One 2017; 12: e0188440

[180] Jansen F, van Uden-Kraan CF, van Zwieten V et al. Cancer survivors' perceived need for supportive care and their attitude towards self-management and eHealth. Support Care Cancer 2015; 23: 1679-1688

[181] Kilbourn KM, Anderson D, Costenaro A et al. Feasibility of EASE: a psychosocial program to improve symptom management in head and neck cancer patients. Support Care Cancer 2013; 21: 191-200

[182] Duffy SA, Ronis DL, Valenstein M et al. A tailored smoking, alcohol, and depression intervention for head and neck cancer patients. Cancer Epidemiol Biomarkers Prev 2006; 15: 2203-2208

[183] Varkey P, Liu YT, Tan NC. Multidisciplinary treatment of head and neck cancer. Semin Plast Surg 2010; 24: 331-334

[184] Dille MF, McMillan GP, Helt WJ et al. A Store-and-Forward TeleAudiology Solution to Promote Efficient Screenings for Ototoxicity during Cisplatin Cancer Treatment. J Am Acad Audiol 2015; 26 : 750-760

[185] Wall LR, Ward EC, Cartmill B et al. Adherence to a Prophylactic Swallowing Therapy Program During (Chemo) Radiotherapy: Impact of Service-Delivery Model and Patient Factors. Dysphagia 2017; 32: 279-292

[186] Hoek PD, Schers HJ, Bronkhorst EM et al. The effect of weekly specialist palliative care teleconsultations in patients with advanced cancer -a randomized clinical trial. BMC Med 2017; 15: 119

[187] Howell D. It is unclear whether specialist palliative care teleconsultation leads to an improvement in patient symptom scores. Evidence Based Nursing 2018; 21: 1-1 
[188] Gomes B, Calanzani N, Curiale V et al. Effectiveness and cost-effectiveness of home palliative care services for adults with advanced illness and their caregivers. Cochrane Database Syst Rev 2013; doi:10.1002/14651858.CD007760.pub2 CD007760

[189] Duursma F, Schers HJ, Vissers KC et al. Study protocol: optimization of complex palliative care at home via telemedicine. A cluster randomized controlled trial. BMC Palliat Care 2011; 10: 13

[190] van Gurp J, van Selm M, Vissers K et al. How outpatient palliative care teleconsultation facilitates empathic patient-professional relationships: a qualitative study. PLoS One 2015; 10: e0124387

[191] Schenker Y, Arnold RM, Bauman JE et al. An enhanced role for palliative care in the multidisciplinary approach to high-risk head and neck cancer. Cancer 2016; 122: 340-343

[192] Penzel T, Schobel C, Fietze I. New technology to assess sleep apnea: wearables, smartphones, and accessories. F1000Res 2018; 7: 413

[193] Maglavera S, Maglaveras N, Lekka I et al. SENSATION remote monitoring system for enabling the "anytime, anywhere" monitoring of patients with selected sleep disorders. Conf Proc IEEE Eng Med Biol Soc 2006; 1: 3869-3872

[194] Singh J, Badr MS, Diebert W et al. American Academy of Sleep Medicine (AASM) Position Paper for the Use of Telemedicine for the Diagnosis and Treatment of Sleep Disorders. J Clin Sleep Med 2015; 11: 1187-1198

[195] Randerath W, Bogel M, Franke C et al. Positionspapier zum Telemonitoring bei schlafbezogenen Atmungsstörungen. Pneumologie 2017; 71: 81-85

[196] Coma-Del-Corral MJ, Alonso-Alvarez ML, Allende M et al. Reliability of telemedicine in the diagnosis and treatment of sleep apnea syndrome. Telemed J E Health 2013; 19: 7-12

[197] Petersen CL, Chen TP, Ansermino JM et al. Design and evaluation of a low-cost smartphone pulse oximeter. Sensors (Basel). 2013: 13: 16882-16893

[198] Garde A, Dehkordi P, Karlen W et al. Development of a screening tool for sleep disordered breathing in children using the phone Oximeter. PLoS One 2014; 9: e112959

[199] Al-Mardini M, Aloul F, Sagahyroon A et al. Classifying obstructive sleep apnea using smartphones. J Biomed Inform 2014; 52: 251-259
[200] Bros JS, Poulet C, Arnol N et al. Acceptance of Telemonitoring Among Patients with Obstructive Sleep Apnea Syndrome: How is the Perceived Interest by and for Patients? Telemed J E Health 2018; 24: 351-359

[201] Woehrle H, Ficker JH, Graml A et al. Telemedicine-based proactive patient management during positive airway pressure therapy: Impact on therapy termination rate. Somnologie (Berl) 2017; 21: 121-127

[202] Sparrow D, Aloia M, Demolles DA et al. A telemedicine intervention to improve adherence to continuous positive airway pressure: a randomised controlled trial. Thorax 2010; 65: 1061-1066

[203] Fox N, Hirsch-Allen A], Goodfellow E et al. The impact of a telemedicine monitoring system on positive airway pressure adherence in patients with obstructive sleep apnea: a randomized controlled trial. Sleep 2012; 35: 477-481

[204] Stepnowsky C], Palau J], Marler MR et al. Pilot randomized trial of the effect of wireless telemonitoring on compliance and treatment efficacy in obstructive sleep apnea. J Med Internet Res 2007; 9: e14

[205] Stepnowsky C, Edwards C, Zamora T et al. Patient perspective on use of an interactive website for sleep apnea. Int J Telemed Appl 2013; 2013: 239382

[206] Frasnelli M, Baty F, Niedermann J et al. Effect of telemetric monitoring in the first 30 days of continuous positive airway pressure adaptation for obstructive sleep apnoea syndrome - a controlled pilot study. J Telemed Telecare 2016; 22: 209-214

[207] Turino C, de Batlle J, Woehrle $\mathrm{H}$ et al. Management of continuous positive airway pressure treatment compliance using telemonitoring in obstructive sleep apnoea. Eur Respir 」 2017; 49

[208] Munafo D, Hevener W, Crocker M et al. A telehealth program for CPAP adherence reduces labor and yields similar adherence and efficacy when compared to standard of care. Sleep Breath 2016; 20: 777-785

[209] Anttalainen U, Melkko S, Hakko S et al. Telemonitoring of CPAP therapy may save nursing time. Sleep Breath 2016; 20: 1209-1215

[210] Woehrle H, Arzt M, Graml A et al. Effect of a patient engagement tool on positive airway pressure adherence: analysis of a German healthcare provider database. Sleep Med 2018; 41: 20-26 